\title{
VI. SMAD-Befehl Nr. 201: Weichenstellung für ein neues Strafprozeßrecht, Justizsteuerung und die politische Strafjustiz (1947-1950)
}

\section{Vorgeschichte und Entstehung von Befehl Nr. 201}

SMAD-Befehl Nr. 201 vom 16. August 1947 führte zu ähnlich weitreichenden Veränderungen im Justizwesen der SBZ wie die Zentralisierung der Justizverwaltung, der Personalaustausch und die grundlegend umstrukturierte Juristenausbildung. Dies gilt nicht nur für den Strafvollzug, sondern auch für das Strafprozeßrecht, die Steuerung der Justiz und die in deutsche Hände übergehende politische Strafjustiz. Dabei ging es in Befehl Nr. 201 prima facie lediglich um die Umsetzung der die Entnazifizierung betreffende Kontrollratsdirektive Nr. 24 sowie der sich primär auf die Bestrafung von Nationalsozialisten beziehende Direktive Nr. 38 in einen gültigen Rechtsakt. Bis zum Erlaß von Befehl Nr. 201 fiel die Bestrafung von NS-Verbrechen ${ }^{1}$ in der SBZ grundsätzlich in die Kompetenz der Besatzungsmacht. Zuständig waren die Militärtribunale, wenngleich sie sich seit Mitte 1946 hauptsächlich gegen politische Oppositionelle sowie Unangepaßte und nur in sehr wenigen Fällen gegen NS-Verbrecher richteten. Die „TerrorElite" des Dritten Reiches war aufgrund von NKWD-Befehl Nr. 0016 vom 11. Januar 1945 weitgehend verhaftet und in Kriegsgefangenenlager in der Sowjetunion abtransportiert worden ${ }^{2}$. In der sowjetischen Zone hatten sich zur Bestrafung von NS-Verbrechern 1945 zudem sogenannte "Volksgerichte“ insbesondere in Sachsen und Brandenburg konstituiert, die auch vereinzelte Urteile aussprachen, ihre Tätigkeit im Zuge der Vereinheitlichung der Gerichtsverfassung aufgrund von SMAD-Befehl Nr. 49 jedoch bald wieder einstellen mußten 3 .

Die einzige Rechtsgrundlage für die Verurteilung von NS-Verbrechen durch deutsche Gerichte in allen Besatzungszonen bot Kontrollratsgesetz Nr. 10 vom

1 In Anlehnung an die überzeugenden Darlegungen von Artzt, Zur Abgrenzung von Kriegsverbrechen und NS-Verbrechen, wird hier durchgehend von NS-Verbrechen und nicht von „Nazi- und Kriegsverbrechen" - so die übliche Bezeichung in DDR-Publikationen - die Rede sein.

2 Vgl. Possekel, Einleitung: Sowjetische Lagerpolitik, in: Sowjetische Speziallager, Bd. 2, S. 65; Befehl Nr. 0016, ebenda, Bd. 2, S. 142-146.

3 Rechtsgrundlage für die Einsetzung eines Volksgerichts in Sachsen war eine Landesverordnung vom 22. 9. 1945; in Brandenburg gab der Landrat von Bernau eine entsprechende Anweisung: siehe Vermerk Kleikamps betr.: Volksgerichte, BAB, DP1 VA Nr. 2, Bl. 48, und Landrat in Bernau an die Amtsgerichte Bernau, Rüdersdorf, Oranienburg, 6. 9. 1945, ebenda, Bl. 17-18; vgl. Benjamin u.a., Geschichte der Rechtspflege 1945-1949, S. $213 \mathrm{f}$., Meyer-Seitz, Verfolgung von NS-Straftaten, S. 24-28; Pohl, Justiz in Brandenburg (Manuskript), S. $14 \mathrm{f}$. 
20. Dezember 1945, das in Artikel III den Satz enthielt: „Für die Aburteilung von Verbrechen, die deutsche Staatsbürger oder Staatsangehörige gegen andere deutsche Staatsangehörige oder Staatenlose begangen haben, können die Besatzungsbehörden deutsche Gerichte für zuständig erklären. " 4 Obwohl die sowjetische Besatzungsmacht eine Reihe teilweise aufsehenerregender Prozesse wie etwa die Ahndung der Euthanasie-Verbrechen in den sächsischen Heilanstalten durch die deutsche Justiz vornehmen ließ ${ }^{5}$, war sie damals - im Unterschied etwa zu den Briten - nicht bereit, alle Verfahren, die von Deutschen an Deutschen verübte NSVerbrechen betrafen, an deutsche Gerichte abzugeben. Zwar blieben in der SBZ die vor allem in den Westzonen weit verbreiteten Schwierigkeiten aufgrund der Durchbrechung des Grundsatzes "nulla poena sine lege" bei diesen Verfahren aus $^{6}$. Die Anwendung von Kontrollratsgesetz Nr. 10 differierte jedoch nicht nur von Land zu Land, sondern teilweise auch innerhalb der einzelnen Länder. So wurden in Brandenburg die Verfahren zunächst vor den Schöffengerichten und erst ab Frühjahr 1947 vor den Strafkammern der Landgerichte oder den Schwurgerichten verhandelt; in Sachsen führten durchweg Schwurgerichte die KRG-10Verfahren durch; in Sachsen-Anhalt und Mecklenburg wurden die schweren Fälle vor die Schwurgerichte, die schwierigeren, in denen weder mit einer hohen Freiheitsstrafe noch mit der Todesstrafe zu rechnen war, vor die Strafkammer und die leichteren vor das Schöffengericht gebracht. Einen Sonderfall bildete Thüringen, wo die Delikte nach Kontrollratsgesetz Nr. 10 immer in Verbindung mit dem betreffenden Straftatbestand des Strafgesetzbuches angeklagt wurden, so daß die Zuständigkeit sich aus den Festlegungen des StGB ergab7 ${ }^{7}$. Insgesamt wurden in der SBZ nach offiziellen Angaben 1945 und 1946 zehn Personen aufgrund von KRG Nr. 10 zum Tode und 119 zu Haftstrafen verurteilt; im Jahre 1947 kamen bis zum Erlaß von Befehl Nr. 201399 Verurteilungen hinzü. Auch danach bildete dieses Gesetz eine Grundlage zur Bestrafung von NS-Verbrechern - das Verfahren wurde jedoch mit dem der 201-Prozesse vereinheitlicht ${ }^{9}$. Zwar gab die Militärverwaltung in einzelnen Verfahren wie dem Dresdner „Ärzteprozeß“ Anweisungen an die deutschen Gerichte; weder sie noch die DJV versuchten indes, die Verfahren nach KRG 10 systematisch zu steuern ${ }^{10}$. Dies sollte sich mit der generellen Übertragung der Ahndung von NS-Verbrechen auf die deutschen Gerichte mit Befehl Nr. 201 grundsätzlich ändern.

4 Amtsblatt des Kontrollrats, Nr. 3, 31. 1. 1946, S. 52. Zur Entstehung des Gesetzes Broszat, Siegerjustiz, S. 484-486.

5 Vgl. dazu Hohmann, Euthanasie-Prozeß.

6 Vgl. Broszat, Siegerjustiz, S. 493, 522-530. In der SBZ sprach sich nur Richard Lange, Universität Jena, gegen die Einführung der rückwirkenden Strafnorm des KRG Nr. 10 aus, ebenda, S. $491 \mathrm{f}$.

7 Siehe Protokoll der Länderkonferenz vom 29. 8. 1947, BAB, DP1 VA Nr. 19, Bl. 33-40.

8 Die offiziellen Zahlen für 1945 und 1946 nach der Gesamtstatistik in: Haltung der beiden deutschen Staaten, S. 32. Für die Zahlen bis zum 16.8. 1947 siehe Benjamin u.a., Geschichte der Rechtspflege 1945-1949, S. 215.

9 Vgl. SMAD-Rechtsabteilung an die Chefs der DJV und der DVdI, 27. 10. 1947, BAB, DP1 VA Nr. 7132, Bl. 50f. Zur Überleitung der Strafsachen nach KRG Nr. 10 in das Verfahren nach Befehl Nr. 201 siehe Meyer-Seitz, Verfolgung von NS-Straftaten, S. 211-214. Wieland, Beitrag der deutschen Justiz, S. 363, erweckt den unzutreffenden Eindruck, als seien nach Erlaß des Befehls Nr. 201 keine Urteile auf Grundlage von KRG Nr. 10 mehr erfolgt.

10 Vgl. Meyer-Seitz, Verfolgung von NS-Straftaten, S. 49, 65. 
Die Entstehungsgeschichte von SMAD-Befehl Nr. 201 ist eng mit den interalliierten Verhandlungen über die Entnazifizierung und die Bestrafung der NS-Verbrecher verknüpft. Als in der SBZ ab Dezember 1946 die weitere Entnazifizierung auf Grundlage der Kontrollratsdirektive Nr. 24 vom 12. Januar desselben Jahres durchgeführt wurde, war dies mit hoher Wahrscheinlichkeit auf das sowjetische Bedürfnis zurückzuführen, sich auf der für Frühjahr 1947 vorgesehenen Außenministerkonferenz in Moskau mit einer vorbildlichen Verwirklichung dieses alliierten Beschlusses zu profilieren und gleichzeitig die Westalliierten auf die Säuberungsdefizite in ihren Zonen hinzuweisen ${ }^{11}$. Auch der erste Anlauf zur Umsetzung der auf der Grundlage des amerikanischen „Befreiungsgesetzes“ vom 5. März $19466^{12}$ vom Alliierten Kontrollrat am 12. Oktober verabschiedeten Direktive Nr. 38 über die „Verhaftung und Bestrafung von Kriegsverbrechern, Nationalsozialisten und Militaristen und Internierung von möglicherweise gefährlichen Deutschen " 13 stand in diesem Zusammenhang. Kontrollratsdirektive Nr. 38 war indes, im Unterschied zu Kontrollratsgesetz Nr. 10, nicht zur Ahndung krimineller Handlungen, sondern eher zur politischen Bewertung und Sühne für Fehlverhalten im Dritten Reich gedacht ${ }^{14}$. Daher machten die Amerikaner auch nicht die ordentlichen Gerichte, sondern Spruchkammern dafür verantwortlich. Die Sowjetunion verfolgte eine andere Linie, indem sie Gerichtsverfahren zur Durchführung der Direktive anstrebte. Bereits im Spätherbst 1946 sprach sich der Leiter der SMAD-Rechtsabteilung gegenüber Polak und Melsheimer dafür aus, vor dem Hintergrund der "schlecht, uneinheitlich und schleppend" arbeitenden Spruchkammern in der amerikanischen Zone die Durchführung der Direktive in der SBZ den ordentlichen Gerichten zu übertragen. Dabei war Karassjow bestrebt, einerseits „von der westlichen Regelung nicht auffallend abzuweichen, andererseits [...] Garantien für ein ordnungsgemäßes, antifaschistisches Funktionieren der Gerichte zu schaffen“. Die Diskussionspartner einigten sich darauf, „daß als erste Instanz die kleine Strafkammer der Landgerichte, als zweite Instanz ein Strafsenat des O[ber]L[andes]-Gerichts tätig werden sollten“, wobei Karassjow jedoch mahnte, bei der Besetzung der Kammern „besondere Sorgfalt obwalten zu lassen“. In einer zweiten Unterredung erklärte sich auch Schiffer am 20. Dezember 1946 mit diesem Verfahren einverstanden. Karassjow erteilte ihm daraufhin den Auftrag, sobald wie möglich einen Verordnungsentwurf zur Durchführung der Kontrollratsdirektive auszuarbeiten. Seine einzige Vorgabe bestand darin, daß die Verfahren den kleinen Strafkammern der Landgerichte „im Bestande eines Richters und zweier Schöffen " übertragen werden sollten ${ }^{15}$.

Ein Entwurf Guskis, der mit Schiffer gründlich beraten wurde, ging am 3. Januar 1947 an die SMAD-Rechtsabteilung. Er sah vor, daß die unterschiedlichen

1 Vgl. Welsh, Revolutionärer Wandel, S. 67 f., Wille, Entnazifizierung, S. 135.

12 Ebenda; das Befreiungsgesetz ist auszugsweise abgedruckt in: Vollnhals, Entnazifizierung, S. 262272.

13 Gedruckt in: Amtsblatt des Kontrollrats, Nr. 11, 31. 10. 1946, S. 184-194 (mit Anhang 195-211).

14 Vgl. Meyer-Seitz, Verfolgung von NS-Straftaten, S. 158.

is Papier ohne Titel, 3. 1. 1947, mit den Vermerken „f. Gen. Ulbricht" und „dikt. von Melsheimer“, SAPMO, NY 4182/1119, BI. 75-77; Aktenvermerk über Besprechung Schiffers mit Karassjow, Bukanow und Jakupow am 20. 12. 1946, BAB, DP1 VA Nr. 11, Bl. 89; vgl. Meyer-Seitz, Verfolgung von NS-Straftaten, S. $162 \mathrm{f}$. 
Fälle je nach Schwere ihrer Belastung im Urteilsverfahren (bei Hauptschuldigen und Belasteten), im Beschlußverfahren (bei Minderbelasteten und Mitläufern) und im vereinfachten Verfahren (bei Mitläufern) behandelt werden sollten. Das Gericht entschied mithin, zu welcher Gruppe der Betroffene gehörte, und legte die Sühnemaßnahmen fest. Für die Beschlußverfahren wurden die kleinen Strafkammern des Landgerichts in der von Karassjow angeordneten Besetzung für zuständig erklärt; bei Urteilsverfahren gegen Hauptschuldige waren ein weiterer Richter und ein weiterer Schöffe hinzuzuziehen. Möglich waren sowohl ein Abwesenheitsverfahren als auch die Einziehung von Vermögen gegen Abwesende und Tote. Als Rechtsmittel sah die DJV die Berufung vor dem Strafsenat des Oberlandesgerichts unter Hinzuziehung von zwei Schöffen vor ${ }^{16}$. Der Entwurf stellte somit den Versuch dar, eine Kontrollratsdirektive unter weitgehender Wahrung rechtsstaatlicher Grundsätze in eine Verordnung für die SBZ umzusetzen. Jakupow bestand am 20. Januar darauf, die Berufung durch die der deutschen Strafprozeßordnung gemäße Revision zu ersetzen, erklärte aber, daß in fünf bis sechs Tagen ein der Instruktion entsprechender Befehl erteilt werde ${ }^{17}$. Auch die SED erfuhr von diesen Bestrebungen, informierte am 31. Januar die Landesvorstände kurz von dem geplanten Verfahren und kündigte den Erlaß der „näheren Verfahrensbestimmungen" durch die DJV an ${ }^{18}$.

Die DJV wartete jedoch vergebens auf ein Signal aus Karlshorst. Es scheint, als wollte die sowjetische Seite vor der nun unmittelbar bevorstehenden Moskauer Außenministerkonferenz angesichts der bereits auf zahlreichen Feldern absehbaren Unvereinbarkeit ihrer Standpunkte mit denen der Westalliierten diese nicht auch im Hinblick auf die Bestrafung der Nationalsozialisten vor vollendete Tatsachen stellen. Die Entnazifizierung bildete dann auch trotz der sowjetischen Kritik an deren Handhabung im Westen einen der wenigen Punkte, über die in Moskau Übereinstimmung erzielt wurde: Sie war im Sinne der Kontrollratsdirektiven Nr. 24 und 38 durch die Zonenkommandeure fortzusetzen, wobei diese auf eine „einheitliche Handhabung" verpflichtet wurden ${ }^{19}$. Die Konferenz beauftragte daraufhin den Alliierten Kontrollrat am 23. April, die Möglichkeit eines Abschlußtermins für die Entnazifizierung zu überprüfen, die Aburteilung von NSVerbrechern zu beschleunigen und die deutschen Behörden zum Erlaß entsprechender Gesetze zur Umsetzung der Direktiven Nr. 24 und 38 zu veranlassen ${ }^{20}$.

Die Sowjetunion erweckte im Kontrollrat zwar noch bis in den August hinein den Eindruck, an dieser gemeinsamen Linie festhalten $z u$ wollen ${ }^{21}$, bereitete jedoch gleichzeitig einen eigenen Befehl zur Durchführung der beiden Direktiven vor. Der wurde am 28. Juli 1947 im Politbüro der KPdSU gebilligt, einen Tag darauf vom stellvertretenden Außenminister Wyschinski an Marschall Sokolowski

i6 Vermerk Guskis, 3. 1. 1947; Entwurf einer Instruktion zur Direktive Nr. 38 des Kontrollrats vom 12. 10. 1946; Chef der DJV an SMAD-Rechtsabteilung, 3. 1. 1947, BAB, DP1 VA Nr. 7817.

17 Vermerk Guskis über Besprechung mit Jakupow, 21. 1. 1947, ebenda.

18 Rundschreiben des SED-Zentralsekretariats an die Landesvorstände, 31. 1. 1947, in: Rößler, Entnazifizierungspolitik, S. 126.

19 Vgl. zur Moskauer Außenministerkonferenz Mai, Alliierter Kontrollrat, S. 400-415, hier 410, Anm. 35.

20 Direktive gedruckt in: Germany 1947-1949, S. 109.

21 Vgl. Welsh, Revolutionärer Wandel, S. 75. 
übersandt und in leicht veränderter Form als SMAD-Befehl Nr. 201 zur Überraschung der westlichen Alliierten am 16. August 1947 erlassen ${ }^{22}$. Weniger überrascht war die SED-Führung. Angesichts der seit Dezember 1946 intensivierten Entnazifizierung aufgrund von Kontrollratsdirektive Nr. 24 drängten sowohl die bürgerlichen Parteien als auch die SED auf ein differenzierteres Vorgehen. Pieck trat im Februar 1947 im „Neuen Deutschland“ dafür ein, „alle wirklich Schuldigen ausfindig zu machen und ihre Verbrechen festzustellen, damit sie von den deutschen Gerichten ihre gerechte Strafe erhalten“, die große Masse der nominellen Parteimitglieder aber durch weitergehende Integrationsmaßnahmen „in den Kampf für die Ausrottung des Hitlerfaschismus und für den Aufbau eines demokratischen Deutschlands“ einzubeziehen ${ }^{23}$. Dieser Doppelstrategie, mit deren Hilfe vor allem die sich seit Winter 1946/47 zuspitzenden innenpolitischen Spannungen abgebaut werden sollten, entsprach auch Befehl Nr. 201, der insofern den Zielen der SED entgegenkam. Daß die SED-Führung, wie bisweilen behauptet, an der Erarbeitung des Befehls beteiligt gewesen sei ${ }^{24}$, läßt sich aufgrund der vorliegenden Quellen zwar nicht belegen; es scheint aber, daß sie über dessen Vorbereitung informiert wurde. Denn bereits am 21./22. Mai 1947 äußerte Pieck auf einer SED-Vorstandssitzung Zweifel darüber, ob für ganz Deutschland einheitliche Richtlinien zur Entnazifizierung gemäß der Direktiven Nr. 24 und 38 gefunden werden könnten, und hielt eine zoneninterne Regelung für wahrscheinlicher ${ }^{25}$. Deutlicher wurde Ulbricht, als er am 1. Juni 1947 auf der Innenministerkonferenz, unter Bezugnahme auf Kontrollratsdirektive Nr. 38 zu bedenken gab: „Es wird also der Zeitpunkt kommen, wo deutsche Gerichte beauftragt werden, Kriegsverbrecher und Verbrecher, die gegen das deutsche Volk gehandelt haben, durch deutsche Gerichte zu bestrafen. Deshalb ist es notwendig, daß unmittelbar die Frage der Säuberung der Gerichte aktiver betrieben wird und man sich dafür interessiert, wer sind die Schöffen? Denn in diesen Gerichten haben die Schöffen die Mehrheit." Die Konferenz beschloß umgehend eine Überprüfung und Schulung der Schöffen, die von der Justiz „unmittelbar und ohne Verzögerung“ eingeleitet werden müßte ${ }^{26}$. Mit Befehl Nr. 201 gab die sowjetische Besatzungsmacht dem Ziel einer inneren Konsolidierung der SBZ Vorrang vor der Aufrechterhaltung der Einigkeit unter den Alliierten in der Entnazifizierungsfrage. Nicht nur bei den "Säuberungen“, sondern auch bei der Bestrafung der NS-Verbrechen durch deutsche Gerichte wollte sie fortan eigene Wege gehen.

22 Rohfassung des Befehls und Begleitschreiben Wyschinskis in: Sowjetische Speziallager, Bd.2, S. 298-301.

23 Piecks Artikel vom 21. 2. 1947 in: Pieck, Reden und Aufsätze, Bd. 2, S. 123-127.

24 So etwa Benser, Zum Prozeß und zu den Wirkungen, S. 706.

25 Auszug aus Piecks Rede in: Rößler, Entnazifizierungspolitik, S. $138 \mathrm{f}$.

26 Gedruckt ebenda, S. 145 f., 142. 


\section{Die Ausführungsbestimmungen von Befehl Nr. 201 für Polizei und Justiz}

Befehl Nr. 201 enthielt kaum Festlegungen zu dem hier ausschließlich interessierenden justitiellen Vorgehen gegen NS-Verbrecher. Nach Absatz 3 waren nur „Kriegsverbrecher, Mitglieder der verbrecherischen Naziorganisationen und führende[.] Persönlichkeiten des Hitlerregimes" von deutschen Gerichten abzuurteilen, nicht aber die „nominellen, nicht aktiven Mitglieder der Nazipartei“. Der Befehl legte des weiteren fest, daß Schuldfeststellung und Bestrafung unter Anwendung der Vorschriften von Kontrollratsdirektive Nr. 38 vorzunehmen seien, die Zuständigkeit des Gerichts sich nach dem Aufenthaltsort des Beschuldigten zu richten habe und die Zentralverwaltungen für Inneres und für Justiz sowie die Länderregierungen die Verantwortung für die Durchführung des Befehls trügen. Ohne säuberliche Abgrenzung der Kompetenzen der Gerichte und der Ermittlungsorgane wurde darin schließlich angeordnet, alle Verbrechensfälle „den deutschen Untersuchungsbehörden zur Bearbeitung zu übergeben "27.

Als sehr viel informativer erwies sich Ausführungsbestimmung Nr. 3 des Befehls, die am 21. August von der SMAD erlassen wurde ${ }^{28}$. Inwieweit bei deren Ausarbeitung auch deutsche Instanzen beteiligt wurden, ist nicht ganz klar ${ }^{29}$; Polak war die Bestimmung bis zu diesem Datum offenbar unbekannt ${ }^{30}$. Sie fixierte erstmals entscheidende Abweichungen vom deutschen Strafprozeßrecht und war somit geeignet, die Rechtsstaatlichkeit der Verfahren nach Befehl Nr. 201 erheblich zu beeinträchtigen. Die „Organe der Innenministerien der Länder" leiteten danach von sich aus die Untersuchung ein, faßten bei Hauptverbrechern einen Beschluß über die Untersuchungshaft und erstellten die Anklageschrift. Eine gerichtliche Voruntersuchung fand nicht statt; das Untersuchungsverfahren lag im wesentlichen in den Händen der Untersuchungsorgane. Die Aufsicht über das Untersuchungsverfahren hatte der Staatsanwalt, dem über dessen Einleitung zu berichten war und der den Haftbeschluß sowie die Anklageschrift zu bestätigen hatte. Wie verfahren werden sollte, wenn der Staatsanwalt die Bestätigung verweigerte, war in der Ausführungsbestimmung nicht festgelegt. Nur für das Gerichtsverfahren selbst galt das deutsche Strafprozeßrecht. Der Befehl legte jedoch - in Abweichung vom GVG - fest, daß die Verhandlungen vor besonderen Strafkammern der Landgerichte durchzuführen waren: Bei Hauptverbrechern mußten diese mit zwei Berufsrichtern und drei Schöffen, bei den anderen zur Anklage kommenden Kategorien mit einem Berufsrichter und zwei Schöffen besetzt werden (später wurden diese als große bzw. kleine Strafkammern bezeichnet). Die Schöffen sollten nicht, wie im GVG vorgesehen, durch das Los bestimmt, sondern

7 Druck in: ZVOBl. 1947, S. 185 f., hier Absatz 3, 7, 8, 9.

28 Druck ebenda, S. 188-191.

29 Wille, Entnazifizierung, S. 167, geht, ohne dies belegen zu können, von einer Beteiligung der DVdI aus. Vorsichtiger ist Meyer-Seitz, Verfolgung von NS-Straftaten, S. 170. Dagegen spricht, daß ein DVdI-Vertreter erst am 23. 8. 1947 im ZS von dem Befehl erfuhr: siehe Bericht Mielkes vom 30. 10. 1947, in: Rößler, Entnazifizierungspolitik, S. 196, und Vermerk betr.: Vorschläge zum Befehl Nr. 201 im Sekretariat Ulbricht-Fechner am 23. 8. 1947, SAPMO, NY 4182/1197, Bl. 145.

30 Polak an Ulbricht, 21. 8. 1947, ebenda, Bl. 146. 
"von den demokratischen Parteien und Organisationen benannt und von den Landesregierungen bestätigt" werden; als Berufsrichter und Schöffen waren nur Personen zuzulassen, „die nicht der NSDAP oder einer ihrer Gliederungen angehört haben und die gemäß ihrer politischen und moralischen Qualitäten geeignet erscheinen, eine demokratische Rechtsprechung zu verwirklichen" - eine Formulierung, die unmißverständlich auf ein im Sinne des Regimes loyales und verläßliches Personal hindeutete. Rechtsstaatlichen Gepflogenheiten entsprach die Möglichkeit, gegen ein Urteil Revision beim Oberlandesgericht einzulegen, wo der Strafsenat „in der üblichen Besetzung“ zu entscheiden hatte. Der Angeklagte erhielt das Recht, einen Verteidiger zur Gerichtsverhandlung hinzuzuziehen; im Ermittlungsverfahren konnten jedoch keine Anwälte eingeschaltet werden, da einer späteren sowjetischen Anweisung zufolge die Akten dem Verteidiger erst mit Eröffnung der Hauptverhandlung zugänglich zu machen waren ${ }^{31}$. Da im Zusammenhang mit Befehl Nr. 201 auch der Ausbau des zuständigen Ermittlungsorgans, der Abteilungen $\mathrm{K} 5$ der Volkspolizei, unter sowjetischer Anleitung erfolgte ${ }^{32}$, ist zu vermuten, daß auch dessen Befugnisse in Richtung derjenigen des MGB in politischen Verfahren erweitert wurden. Auch die formelle Machtfülle der Staatsanwaltschaft im Ermittlungsverfahren ist eindeutig dem sowjetischen Rechtssystem entnommen ${ }^{33}$. Da gleichzeitig wesentliche Elemente des deutschen Prozeßrechts erhalten blieben, ist dem damaligen thüringischen Generalstaatsanwalt Friedrich Kuschnitzky bei seiner Bezeichnung des Verfahrens als einer „Synthese zwischen deutscher und russischer Rechtsauffassung "34 durchaus zuzustimmen.

Auf deutscher Seite wurde als erstes nicht die DJV, sondern am 21. August Polak über Befehl Nr. 201 und dessen Bedeutung für die Justiz informiert - ein Hinweis darauf, daß die SMAD dessen Umsetzung vor allem über die SED bewerkstelligen wollte ${ }^{35}$. Die SED-Spitze berief zwei Tage später eine erste kleinere Vorbesprechung ins Büro von Ulbricht und Fechner mit jeweils einem Genossen aus der DVdI und aus der DJV ein. Danach wurde als erstes am 25. August eine Konferenz der Innenminister abgehalten, an der für die SMAD Jakupow teilnahm. In diesen Tagen ernannte das DVdI-Präsidium Vizepräsident Erich Mielke zum Hauptverantwortlichen für die Durchführung von Befehl Nr. 20136. Erst nachdem die Organe der Inneren Verwaltung informiert worden waren und erste Schritte zur Umsetzung des Befehls unternommen hatten, wurden die Landesjustizminister, die Generalstaatsanwälte und die Oberlandesgerichtspräsidenten am 29. August zu einer Konferenz in die DJV eingeladen. Auch diesmal äußerte sich Jakupow zu den Fragen der "Gerichtspraxis“ in den Verfahren nach Befehl Nr. 201 und wiederholte dabei im wesentlichen die Einzelheiten der Ausführungsbestimmung Nr. 3. Hinzu kamen einige Präzisierungen, die im wesentlichen

31 So Jakupow bei der Länderkonferenz am 14./15. 11. 1947, BAB, DP1 VA Nr. 6332.

32 Vgl. dazu das nächste Unterkapitel.

33 Vgl. Hellbeck, Staatsanwaltschaft, S. 77; Karew, Sowjetische Justiz, S. 121, 123.

34 Kuschnitzkys Äußerung in: Protokoll über die Konferenz mit den Vertretern der Justizministerien am 14./15. 11. 1947, BAB, DP1 VA Nr. 6332.

35 Polak an Ulbricht, 21. 8. 1947, SAPMO, NY 4182/1197, Bl. 146.

36 Bericht Mielkes vom 30. 10. 1947, in: Rößler, Entnazifizierungspolitik, S. 196; Vermerk betr.: Vorschläge zum Befehl Nr. 201 im Sekretariat Ulbricht-Fechner am 23. 8. 1947, SAPMO, NY 4182/ 1197, Bl. 145. 
die Aufgaben und Befugnisse des Staatsanwalts gegenüber den Untersuchungsorganen betrafen. So stellte er klar, daß die „Aufsicht" des Staatsanwalts sich nur auf Einleitung und Kontrolle des jeweils anstehenden Untersuchungsvorgangs bezog, nicht aber ein allgemeines Kontroll- und Eingriffsrecht in die Angelegenheiten der inneren Verwaltung umfasse. Die Bestätigung des Haftbeschlusses durch den Staatsanwalt war nach Jakupows Ausführungen weitgehend eine Formalität, da Hauptverbrecher ohnehin verhaftet werden mußten und bei den anderen Verbrechern das Ermessen der Ermittlungsorgane entscheidend war. Im Hinblick auf die von den Untersuchungsorganen anzufertigende Anklageschrift räumte er dem Staatsanwalt das Recht ein, diese „im einen oder anderen Falle“ nicht zu bestätigen. Bei der Feststellung einer mangelhaften Untersuchung oder unvollständiger Unterlagen solle der Staatsanwalt die Sache zur Ergänzung zurückgeben; eine trotz einwandfreier Untersuchung mangelhafte Anklageschrift könne dieser neu zusammenstellen. Schließlich sei der Staatsanwalt auch befugt, die Frist für die Untersuchung festzusetzen, für die in Ausführungsbestimmung Nr. 3 keine präzise Bestimmung existierte ${ }^{37}$. Jakupow schrieb mit seinen Anweisungen somit die eingeschränkten Befugnisse des Staatsanwalts gegenüber den Untersuchungsorganen fest.

Die Vorrangstellung der DVdI bei der Durchführung von Befehl Nr. 201 wird auch daran deutlich, daß im ersten Abschnitt von Ausführungsbestimmung Nr. 3 ihr die Aufgabe übertragen worden war, eine weitere Durchführungsvorschrift zu erlassen $^{38}$. Jakupow forderte am 29. August die DJV zwar dazu auf, an deren Herausgabe mitzuarbeiten ${ }^{39}$; die DVdI jedoch ließ ihren Entwurf, ohne die DJV zu konsultieren, bereits am selben Tag der Innenverwaltung und Rechtsabteilung in Karlshorst zukommen, was Schiffer erst im nachhinein erfuhr ${ }^{40}$. Da nun die Chance einer Kooperation vertan war, unterbreitete die DJV ihren Entwurf der SMAD-Rechtsabteilung am 9. September, freilich nicht ohne tags zuvor der DVdI ein Exemplar zukommen zu lassen. Bei der Vorlage des auf den Äußerungen Jakupows basierenden DJV-Entwurfs in Karlshorst durch Winkelmann ging es wieder um die Rechte des Staatsanwalts gegenüber den Untersuchungsorganen. Jakupow revidierte dabei seine Aussage vom 29. August, daß dieser die Fristen für die Untersuchung setze; es sei vielmehr „beabsichtigt, der Untersuchungsbehörde allgemeine Fristen zu setzen" ${ }^{41}$. Winkelmann akzeptierte dies zwar, wollte aber die Eingriffsrechte des Staatsanwalts erweitern und fragte, ob dieser „notfalls auch in die Vernehmungen oder durch Teilnahme an den Vernehmungen in die Untersuchung eingreifen dürfe" ${ }^{42}$. Bei der folgenden Unterredung am 13. September wurde klar, daß die DJV mit diesen Bestrebungen nicht durchkam. Denn anstelle des ursprünglichen Textes, demzufolge der Staatsanwalt „zur Erteilung bindender Weisungen an die Untersuchungsbehörden" befugt sei, wies Nikolajew an, den

37 Protokoll der Konferenz vom 29. 8. 1947, BAB, DP1 VA Nr. 19, Bl. 10-13.

38 Siehe die Anmerkung zu Absatz 1 der Ausführungsbestimmung Nr. 3, in: ZVOBl. 1947, S. 188.

39 Protokoll der Konferenz vom 29. 8. 1947, BAB, DP1 VA Nr. 19, Bl. 7.

40 Bericht Mielkes vom 30.10. 1947, in: Rößler, Entnazifizierungspolitik, S. 197; Chef der DJV an DVdI, 8. 9. 1947, BAB, DO1/7 Nr. 424, Bl. 30.

41 Die Frist wurde in den Ausführungsbestimmungen der DVdI vom 12.9.1947 auf 2 Monate festgesetzt: vgl. Winkelmann, Aufgaben der Staatsanwälte, S. 169.

42 Vermerk Winkelmanns, 9. 9. 1947, BAB, DP1 VA Nr. 6203, Bl. 63. 
Passus folgendermaßen zu formulieren: „Er ist zur Erteilung von Weisungen an die Untersuchungsbehörde in der einzelnen Ermittlungssache befugt. " ${ }^{43}$ Dies schloß eine generelle Unterstellung der Kriminalpolizei unter die Staatsanwaltschaft aus und erlaubte der K 5, weitgehend unabhängig von der Justiz zu agieren. Die DJV mußte sich mit dieser und einer Reihe anderer Umformulierungen einverstanden erklären ${ }^{44}$, bevor Schiffer den Erlaß über die „Aufgaben der Gerichte und Staatsanwälte" ${ }^{45}$ am 18. September unterzeichnen konnte. Auffällig ist, daß Kritikpunkte aus der DVdI ${ }^{46}$ an dem ursprünglichen DJV-Entwurf in der Endfassung des Erlasses fast alle berücksichtigt worden waren - ein Indiz dafür, daß der Innenverwaltung in dieser Frage ein weitaus größeres Gewicht zukam als der Justizverwaltung.

Während die DVdI durch die rechtzeitige Information Schiffers die Chance erhielt, auf den DJV-Erlaß Einfluß zu nehmen, bekam die Justizverwaltung den Entwurf der Durchführungsvorschrift trotz Nachfrage bei der SMAD-Rechtsabteilung nicht zu sehen ${ }^{47}$. Innenverwaltung und Rechtsabteilung der SMAD genehmigten die am 29. August von der DVdI eingereichten „Ausführungsbestimmungen über die Regelung der Feststellung und Registrierung von Hauptverbrechern, Verbrechern und Minderbelasteten", die am 12. September in Kraft traten ${ }^{48}$. Darin war unter anderem - auf Wunsch der SMAD-Rechtsabteilung - festgelegt worden, daß der Präsident des Landgerichts nach erfolgtem Urteil der DVdI, dem Landesinnenminister und der untersuchungsführenden Polizeidienststelle zu berichten hatte ${ }^{49}$. Um möglichst weitgehende Einblicke in die Tätigkeit der Gerichte zu bekommen, forderte Mielke am 30. September außerdem, daß die aufsichtsführenden Staatsanwälte bei Abänderung oder Neufassung der Anklageschriften die untersuchungsführende Polizeidienststelle von den vorgenommenen Veränderungen in Kenntnis setzen sollten. Darüber hinaus schlug er vor, eine Kommission aus DVdI- und DJV-Vertretern zu bilden, „um die reibungslose Zusammenarbeit zwischen aufsichtsführenden Staatsanwälten und Polizeidienststellen zu gewährleisten", und bat um Mitteilung der Namen der bei den zu errichtenden Strafkammern nach Befehl Nr. 201 eingesetzten Richtern und Staatsanwälten. Die DJVSpitze entsprach allen Forderungen; man kam lediglich überein, nicht die Namen aller Richter und Staatsanwälte, sondern nur die der Gerichtsvorsitzenden mitzuteilen ${ }^{50}$.

Sowohl die Ausführungsbestimmungen der SMAD als auch die ebenfalls unter strikter sowjetischer Aufsicht angefertigten Durchführungsvorschriften der DVdI und der DJV schwächten die Justiz in den Verfahren nach Befehl Nr. 201

43 Vermerk Winkelmanns, 15. 9. 1947, ebenda, Bl. 53.

44 Siehe Vermerk über Unterredung mit Jakupow am 15. 9. 1947 mit Anlage Nr. 2, ebenda, Bl. 54, 52.

45 Gedruckt in: ZVOBl. 1947, S. 191-194.

46 Abteilung P, Referat P7, an Mielke, 13. 9. 1947, BAB, DO1/7 Nr. 424, Bl. 32.

47 Vermerk Winkelmanns, 9. 9. 1947, BAB, DP1 VA Nr. 6203, Bl. 63.

48 Ausführungsbestimmungen in: BAB, DO1/7 Nr. 69, Bl. 34 f., o.D.; zum Datum siehe Vermerk über Unterredung mit Jakupow am 15. 9. 1947, BAB, DP1 VA Nr. 6203, Bl. 53.

49 Ebenda.

so Vermerk Winkelmanns, 3. 10. 1947, ebenda, Bl. 29; das Schreiben Reschkes vom 25.9. 1947 ebenda, Bl. 37. Infolge der Besprechung verschickte die DJV am 3. 10. 1947 eine Rundverfügung, der die Ausführungsbestimmungen der DVdI vom 12.9. 1947 für die Richter und Staatsanwälte beigefügt waren, BAB, DO1/7 Nr. 69, Bl. 89-92. 
erheblich. Die Entstehungsbedingungen zeigen, daß die DJV angesichts der sowjetischen Vorgaben und der übermächtigen Stellung der DVdI keine Chance besaß, etwa durch eine großzügige Auslegung der Rechte des Staatsanwalts, die Justizorgane am Untersuchungsverfahren stärker zu beteiligen. Sie mußte vielmehr hinnehmen, daß die Innenverwaltung durch ihre eingehenden Informationen über die Tätigkeit der Staatsanwälte und der Gerichte letztlich auch die Möglichkeit erhielt, diese zu beeinflussen.

\section{Akteure und Methoden der Justizsteuerung in den Verfahren nach Befehl Nr. 201}

Mit Befehl Nr. 201 übergab die sowjetische Besatzungsmacht die Rechtsprechung auf einem für ihre Nachkriegspolitik zentralen Gebiet der deutschen Gerichtsbarkeit. Jedoch ergab sich nicht nur daraus das nachhaltige sowjetische Interesse an der Durchführung dieser Prozesse. Wie der Vortrag eines SMAD-Offiziers von Ende August/Anfang September 1947 zeigt, hatte man den Befehl auch erlassen, „um die Gerichte schwimmen zu lassen und in der Praxis festzustellen, was sie für Schwimmer sind". Anhand der Rechtsprechung in diesen Prozessen wollte Karlshorst also auch überprüfen, inwieweit die deutschen Gerichte inzwischen in der Lage waren, NS-Straftäter nach sowjetischen Vorstellungen abzuurteilen. Dies implizierte eine enge Überwachung der deutschen Justiz auf diesem Gebiet, „damit es dabei keine Fehler und keine Schädlichkeitsakte, die gegen die Interessen der Demokratisierung Deutschlands gerichtet sind, gibt“. „Die Rechtsabteilung und die Abteilung für Inneres“, so der sowjetische Offizier weiter, „müssen die richtige Durchführung des Befehls Nr. 201 sichern und auch berücksichtigen, daß wir in der Justiz offensichtlich Unordnung haben, daß in der Landesjustizverwaltung und in den örtlichen Justizorganen sehr viele reaktionäre Elemente sitzen". ${ }^{51}$ Die deutschen Gerichte waren zwar mit den 201-Verfahren auf ihre Zuverlässigkeit zu überprüfen und gegebenenfalls als „reaktionär" zu entlarven; sie bedurften jedoch, um ungewollte Entscheidungen zu vermeiden, der engen Beaufsichtigung und Kontrolle durch die zuständigen SMAD-Abteilungen und ihre deutschen Hilfsorgane. Dem Steuerungswillen der SMAD-Rechtsabteilung kam dabei entgegen, daß sie für die authentische Auslegung des Befehls zuständig war und bei Zweifelsfällen von den deutschen Instanzen konsultiert wurde.

Auf deutscher Seite nahm die SED-Führung, die sich anschickte, die Justiz „, in die Hand [zu] bekommen" 52 , die sowjetische Herausforderung an und stellte sie in einen engen Zusammenhang mit ihrem justizpolitischen Konzept. „Der Befehl 201“, so Fechner auf der Parteivorstandstagung am 14./15. Januar 1948, „ist ein Prüfstein für den bisher erreichten Grad der Demokratisierung der Justiz." $" 53 \mathrm{Be}$ -

51 Undatiertes Redemanuskript unbekannter sowjetischer Herkunft, in: Badstübner/Loth, PieckAufzeichnungen, S. 170.

52 So Melsheimer vor dem Ausschuß für Rechtsfragen beim ZS der SED, 3./4. 1. 1948, SAPMO, DY 30 IV 2/1.01/70, Bl. 233.

53 Protokoll der Tagung des SED-Parteivorstands am 14./15. 1. 1948, SAPMO, DY 30 IV 2/1/38, Bl. 121. Vgl. auch die Äußerung Benjamins auf der Justizkonferenz vom 11./12.6. 1948, BAB, 
fehl Nr. 201 bot damit der Parteiführung nicht nur die Möglichkeit, die Konformität der Justiz mit ihrer Politik zu überprüfen, sondern auch eine weitere Legitimation für die Durchsetzung ihrer politischen Zielsetzungen im Hinblick auf den Rechtsstab und die Rechtsprechung. Es war daher nur konsequent, wenn die SED-Spitze nach Erlaß des Befehls den Parteiapparat und ihre herausgehobene Stellung als politisches Koordinationszentrum in der SBZ für justizsteuernde Maßnahmen nutzte. Sowohl von der SED-Führung als auch von der SMAD-Innenverwaltung angeleitet, erwies sich die DVdI als weitere in diesem Zusammenhang interessierte Steuerungsinstanz. Erich Mielke sah in dem Befehl ebenfalls einen „Prüfstein dafür, inwieweit die deutschen Organe in der Lage sind, diese Aufgabe [der Bestrafung der NS-Verbrecher] durchzuführen, ferner dafür, inwieweit die Bevölkerung den neuen demokratischen Aufbau, der besonders von der Sozialistischen Einheitspartei gefördert wird, als richtig anerkennt und somit auch von der Richtigkeit überzeugt ist, auch den letzten aktiven Faschisten seiner gerechten Strafe zuzuführen“. Befehl Nr. 201 bildete für Mielke somit „eine Teilfrage des Kampfes um die Festigung der demokratischen Macht “54. Unter dieser Voraussetzung betrachtete er die Gerichte lediglich als Ausführungsorgane der Polizei: „Es muß erreicht werden“, so formulierte er auf einer Tagung der DVdI, „daß die Justizorgane ebenfalls spuren. "55 Die DJV war als Steuerungsinstanz abhängig von der zuständigen SMAD-Abteilung, der SED-Führung und der DVdI aber eindeutig nachgeordnet. Obgleich sie als die im engeren Sinne zuständige Zentralverwaltung angesehen werden muß, kam ihr bei der Steuerung der Rechtsprechung eher die Rolle eines Transmissionsriemens der Militärverwaltung zu. Ihr Handlungsspielraum war vor allem dadurch begrenzt, daß die SMAD-Rechtsabteilung sie in dieser Frage am kurzen Zügel führte; hinzu kam ab Mitte 1948, daß die kommunistischen Führungskräfte in der DJV den Kurs der Zentralverwaltung bestimmten und sich als treue Vollstrecker der SED-Politik erwiesen.

Die Übermittlung zentraler Vorgaben bei der Lenkung der Justiz in den Verfahren nach Befehl Nr. 201 erfolgte über bereits erprobte Kommunikationsschienen. Die SED versuchte, über Rundschreiben an die Landesvorstände Einfluß zu nehmen, und setzte darauf, daß die Stellungnahme ihres rechtspolitischen Beirats zur Durchführung der Verfahren vom 4. Januar 1948 über dessen Mitglieder verbreitet und beachtet wurde ${ }^{56}$. Mielke wies auf der Innenministerkonferenz vom 29./ 30. November 1947 darauf hin, daß die zuständigen Vertreter in den Innenministerien und Justizministerien eines jeden Landes dem SED-Landessekretariat über die Durchführung des Befehls zu berichten hatten, woraus sich nicht nur ein entsprechender Informationsstand, sondern auch Einflußmöglichkeiten ergaben ${ }^{57}$. Die DVdI konnte die Untersuchungsorgane aufgrund der zentralisierten Struktur

DP1 VA Nr. 264, Bl. 19: „Die Durchführung von Befehl 201 sei eine Probe für die Demokratisierung von Polizei und Justiz."

54 Bericht Mielkes vom 30. 10. 1947, in: Rößler, Entnazifizierungspolitik, S. 205.

55 Protokoll der Tagung über den Befehl $201 \mathrm{am}$ 22. 12. 1947 bei der DVdI, ebenda, S. 239.

56 Siehe u.a. Rundschreiben des Zentralsekretariats Nr. 21/47, 10.9. 1947, in: Rößler, Entnazifizierungspolitik, S. 168-172. Das ZS stimmte am 8.1. 1948 der Stellungnahme des rechtspolitischen Beirats zu, SAPMO, DY 30 IV 2/2.1/162, TOP 4, Anlage Nr. 3.

57 Aus dem Protokoll der Innenministerkonferenz am 29./30.11. 1947, in: Rößler, Entnazifizicrungspolitik, S. 213. 
der Polizei von Berlin aus lenken und behandelte das Thema darüber hinaus auf zahlreichen Besprechungen mit den Innenministern oder mit den für die Durchführung des Befehls zuständigen Polizeifunktionären ${ }^{58}$. Außerdem nutzte Mielke seine Anwesenheit bei den zentralen Konferenzen der DJV von August und November 1947 und bei der Justizkonferenz vom Juni 1948, um auch auf die Zusammensetzung der Gerichte und die Rechtsprechung Einfluß zu nehmen.

Zur Justizsteuerung nutzte die SMAD-Rechtsabteilung 1947/48 Konferenzen auf zonaler und auf Landesebene sowie Rundverfügungen der DJV an die Landesjustizministerien. Unmittelbar nach Erlaß des Befehls fand eine Vielzahl von Tagungen statt, die die Justiz nicht nur mit den neuen Vorschriften bekannt machen sollten. So kritisierte Jakupow etwa auf der Konferenz vom 29. August 1947, daß die deutschen Gerichte in den Verfahren nach Kontrollratsgesetz Nr. 10 in der Regel zu milde geurteilt hätten, und verlangte verklausuliert eine härtere Bestrafung in den Prozessen nach Befehl Nr. 201. In Zukunft müßten „die Justizministerien der Länder den Gerichten Hinweise geben und kontrollieren, daß die Direktive Nr. 38 richtig durchgeführt wird" ${ }^{59}$. Eine erste Gelegenheit, diese Anweisung weiterzugeben, ergab sich auf den daraufhin im September durchgeführten Landeskonferenzen zu Befehl Nr. 201. Inwieweit den Anordnungen Folge geleistet worden war, konnten DJV und SMAD-Rechtsabteilung auf der Länderkonferenz am 14./15. November überprüfen, auf der die Besetzung der Strafkammern, die Zusammenarbeit zwischen Justiz und Untersuchungsorganen und Zweifelsfragen bei der Anwendung der Rechtsvorschriften im Vordergrund standen. Wieder hatte Jakupow Gelegenheit zu kritischen Äußerungen, deren justizsteuernde Absicht unverkennbar war ${ }^{60}$. Auf der zentralen Justizkonferenz vom Juni 1948 schließlich stand das Referat Melsheimers über den „Kampf der deutschen Justiz gegen die Naziverbrecher “ im Vordergrund. Die Wirkungen seiner Äußerungen etwa über die durchweg als zu gering kritisierte Strafzumessung sind dabei um so höher zu veranschlagen, als er seinen Vortrag - der im übrigen auch in der „Neuen Justiz" abgedruckt wurde - auf den nachfolgenden Länderkonferenzen wiederholte und damit fast alle Justizjuristen in der SBZ erreichte ${ }^{61}$. Der Kontrolle der Rechtsprechung nach Befehl Nr. 201 diente die Berichterstattung der Länder an die DJV. Die wiederum mußte nicht nur die SMAD-Rechtsabteilung, sondern auch den „sowjetischen Verbindungsoffizier“ im Hause - also die sowjetische Geheimpolizei - und zeitweise auch das „Informationsbüro" der SMAD"2 in BerlinLichtenberg unterrichten. Anfangs waren Wochenmeldungen anzufertigen und an den Verbindungsoffizier weiterzugeben ${ }^{63}$; nachdem in der SMAD-Rechtsabteilung registriert worden war, daß der ausufernde Papierkrieg die Arbeit der

58 Siehe vor allem die Protokolle dieser Besprechungen ebenda, S. 191-195, 212-217, 221-243.

59 Protokoll der Konferenz vom 29. 8. 1947, BAB, DP1 VA Nr. 19, Bl. 15 f.

60 Protokoll der Länderkonferenz am 14./15. 11. 1947, BAB, DP1 VA Nr. 6332.

61 Referat Melsheimers in: Die Juristenkonferenzen, S. 126-131. Vor dem Hintergrund der weiten Verbreitung von Melsheimers Äußerungen ist es m.E. verfehlt, diese als „unverbindliche Form der Anleitung" abzutun (so Meyer-Seitz, Verfolgung von NS-Straftaten, S. 205).

62 Vermerk Benjamins, 18. 11. 1947, BAB, DP1 VA Nr. 6204, Bl. 146.

${ }_{63}$ Vermerk Benjamins, 17. 11. 1947, BAB, DP1 VA Nr. 580, Bl. 73. 
Justiz beeinträchtigte, ließ man es ab Januar 1948 bei monatlichen Berichten bewenden ${ }^{64}$.

Eines der wichtigsten justizsteuernden Mittel in den 201-Prozessen stellte von Anfang an die ,richtige' Besetzung der Strafkammern dar. Um dies sicherzustellen, legte Jakupow am 29. August fest, daß die Richter und Staatsanwälte von den Justizministerien und Oberlandesgerichtspräsidenten nach der Vorgabe von Ausführungsbestimmung Nr. 3 auszuwählen, aber nur ,im Einvernehmen mit den SMA's der einzelnen Länder" $z$ u bestellen seien ${ }^{65}$. Auch die Vorauswahl sollte aber nicht allein der Justiz überlassen werden. Am 21. August, unmittelbar nach seiner Unterredung über Befehl Nr. 201 mit Bukanow und Jakupow hielt Polak fest: „Auch die ordentlichen Richter der Kammern werden namentlich ernannt. Es ist eine Vorarbeit der Personalpolitischen Abteilung [der SED] erforderlich bei der Durchführung dieser Benennungen. Man wies darauf hin, daß man sehr auf die Unterstützung der antifaschistischen Parteien dort rechnet." ${ }^{66}$ Während die Kooperation mit CDU und LDP allenfalls bei der Benennung der Schöffen erwünscht war, blieb die Vorauswahl der Berufsrichter insbesondere der SED vorbehalten, die in der Besetzung der Strafkammern mit SED-konformen Justizjuristen eine wesentliche Voraussetzung für das Bestehen der „Bewährungsprobe“ in Gestalt von Befehl Nr. 201 sah. Die Berufsrichter waren daher entweder aus den eigenen Reihen oder aus den Absolventen der Richterschulen zu rekrutieren ${ }^{67}$. Die SED-Landesvorstände wurden in diesem Rundschreiben angehalten, auf eine entsprechende Besetzung der Gerichte zu achten; „für die Auswahl und Bestätigung dieser Richter“ waren nicht nur die Justizminister, sondern auch die Innenminister verantwortlich ${ }^{68}$. Das Ziel dieser weniger von der Justiz, sondern mehr von anderen Instanzen vorgenommenen Einflußnahmen wurde am offensten von Erich Mielke formuliert: „Es muß erreicht werden im Zusammenhang mit den anderen zuständigen Stellen, daß die Zusammensetzung so erfolgt, daß zum mindesten nach vorherigen [sic] Ermessen eine richtige Schuldfeststellung sichergestellt wird."69

Da nicht genügend Justizjuristen mit einem SED-Parteibuch zur Verfügung standen, ließ sich das Ziel, die Strafkammern nach Befehl Nr. 201 fast ausschließlich mit Richtern und Staatsanwälten aus der eigenen Partei zu besetzen, in den ersten Monaten nach Erlaß des Befehls nicht verwirklichen. Freilich gehörten bereits im November 194751 von 86 Richtern und 31 von 44 Staatsanwälten in den Strafkammern und Berufungsinstanzen der SED an; im Februar 1948 waren 55 der 98 Richter und 46 der 50 Staatsanwälte SED-Mitglieder, so daß die Einheitspartei insgesamt 68 Prozent der Berufsjuristen in diesen Verfahren stellte ${ }^{70}$. Bis

64 Mitteilung an den sowjetischen Verbindungsoffizier, 7. 1. 1948, ebenda, B1. 83.

65 Protokoll der Konferenz vom 29. 8. 1947, BAB, DP1 VA Nr. 19, Bl. 7 f.

66 Polak an Ulbricht, 21. 8. 1947, SAPMO, NY 4182/1197, Bl. 146.

67 Betr.: Vorschläge zum Befehl Nr. 201 bei der Besprechung im Sekretariat Ulbricht-Fechner am 23. 8. 1947, 25. 8. 1947, ebenda, Bl. 145.

68 Rundschreiben Nr. 21/4 des Zentralsekretariats, 10. 9. 1947, in: Rößler, Entnazifizierungspolitik, S. 169.

69 Stenographische Niederschrift über die Tagung des Ausschusses für Rechtsfragen beim ZS, 3. 1./ 4. 1. 1948, SAPMO, DY 30 IV 2/1.01/70, Bl. 109.

70 Zahlen errechnet nach den Aufstellungen in: BAB, DP1 VA Nr. 6204, Bl. 148, und Vermerk, 8. 2. 
zum Januar 1948 waren Benjamin zufolge in allen Ländern außer Thüringen zwei Drittel bis drei Viertel aller Kammervorsitzenden und fast alle aufsichtsführende Staatsanwälte SED-Genossen ${ }^{71}$. In Einzelfällen besetzten jedoch auch LDP- und CDU-Angehörige leitende Positionen der Strafkammern, was die DVdI in einem internen Schreiben beklagte ${ }^{72}$. Die Anzahl der Volksrichter lag mangels Masse anfangs weitaus niedriger als die der SED-Juristen: Nach einer Aufstellung vom 10. September 1947 hatten lediglich 26 von 100 mit der Durchführung der Prozesse nach Befehl Nr. 201 betraute Richter und Staatsanwälte einen Richterlehrgang absolviert ${ }^{73}$. Wenngleich diese Zahlenangaben unvollständig waren und sich der Anteil der Volksrichter innerhalb der nächsten Monate erhöht haben dürfte, erscheint Hilde Neumanns Aussage von Ende Oktober, in diesen Verfahren seien „zu über 50\% “ Volksrichter eingesetzt, als übertrieben”4. Der SED gelang es zwar nicht im ersten Anlauf, die 201-Kammern fast auschließlich mit Personen ihres Vertrauens zu besetzen; im Verlauf des Jahres 1948, vor allem nach Beendigung des dritten Volksrichterlehrgangs im September, rückten fast ausschließlich Lehrgangsabsolventen in die Strafkammern nach, die aufgrund von Weisungen der DJV in zunehmendem Maße auch als Kammervorsitzende eingesetzt wurden 75 .

Größere Einflußmöglichkeiten besaß die SED im Hinblick auf die Auswahl der Laienrichter, da laut Ausführungsbestimmung Nr. 3 die Parteien und Massenorganisationen die Schöffen benannten, die dann von der Landesregierung zu bestätigen waren. Der Bedeutung der Schöffen, die sowohl in den großen als auch in den kleinen Strafkammern die Mehrheit stellten, war sich die SED von Anfang an bewußt. Die Benennung der Kandidaten erfolgte aufgrund einer Vereinbarung zwischen dem Landesblockausschuß und den Vertretern der Massenorganisationen, so daß ein Mitspracherecht der SED auch bei den Kandidaten der anderen Parteien gesichert war. Kandidaten aus den eigenen Reihen waren von dafür gebildeten Landeskommissionen zu benennen, die sich aus je einem Vertreter der Landespersonalabteilung, des Innenministeriums und des für das Landgericht zuständigen Kreissekretariats zusammensetzen sollten. Um eine SED-Mehrheit unter den Laienrichtern zu sichern, gab das Zentralsekretariat zudem die Anweisung festzustellen, „wieviel [sic] Schöffen gebraucht werden, und daß dann die doppelte Anzahl gestellt wird"76. Auch die SMAD versuchte, die Zusammensetzung

1948, BAB, DP1 VA Nr. 819, Bl. 21. Nach der Aufstellung von Wille, Entnazifizierung, S. 188, gehörten von 102 Richtern und Staatsanwälten 64 der SED an.

71 Stenographische Niederschrift über die Tagung des Ausschusses für Rechtsfragen beim ZS, 3. 1./ 4. 1. 1948, SAPMO, DY 30 IV 2/1.01/70, B1. 127.

72 Vgl. Wille, Entnazifizierung, S. $188 \mathrm{f}$.

73 Zahlen nach DJV an SMAD-Rechtsabteilung, 10. 9. 1947, BAB, DP1 VA Nr. 840/1, Bl. 70-77. Diese Angaben sind unvollständig, da der DJV weder die Besetzungslisten von allen Berufungssenaten bei den Oberlandesgerichten noch die Namen aller beisitzenden Richter vorlagen.

74 Besprechung über den Befehl 201, 30. 10. 1947, in: Rößler, Entnazifizierungspolitik, S. 193.

75 Siehe die von der DJV der SMAD-Rechtsabteilung seit September 1948 übermittelten Veränderungsmeldungen in: BAB, DP1 VA Nr. 840/2. Nach den Vergleichszahlen für Sachsen-Anhalt, Brandenburg, Thüringen und Mecklenburg waren dort insgesamt bis zum 26.1. 194910 Volksrichter Vorsitzende von kleinen oder großen Strafkammern nach Befehl Nr. 201, BAB, DP1 SE Nr. 3475. Ein signifikantes Übergewicht in den 201-Kammern hatten sie erst 1951/52: In einer Akte mit Personalunterlagen eines Teils der Richter in diesen Strafkammern waren 35 von 49 Volksrichter (BAB, DP1 SE Nr. 445).

76 Rundschreiben Nr. 21/4 des Zentralsekretariats, 10. 9. 1947, in: Rößler, Entnazifizierungspolitik, S. $169 \mathrm{f}$. 
der Schöffen zu beeinflussen. Wie der brandenburgische Innenminister Bernhard Bechler dem Landesblockausschuß in Potsdam mitteilte, seien vor dem Hintergrund der ,aufgrund der Einwirkung der Schöffen“ zu milden Urteile nach Kontrollratsgesetz Nr. 10 „auf besonderen Wunsch des Vertreters der SMAD Karlshorst“ diese nun vornehmlich aus dem Kreis der „Opfer des Faschismus“ zu rekrutieren, „da nur sie das notwendige Maß an innerer Einstellung haben, um wirklich scharf gegen diese Verbrecher vorgehen zu können"77. Zur Anleitung der Schöffen fügte die SED-Zentrale ihrem Rundschreiben vom 10. September 1947 eine Anlage bei, in der nicht nur über die Besonderheiten der Verfahren nach Ausführungsbestimmung Nr. 3 informiert, sondern unter anderem auch darauf hingewiesen wurde, daß bei diesen Verfahren kein individueller Schuldnachweis zur Verurteilung erforderlich sei ${ }^{7}$. Eine Steuerungsfunktion kam auch der Schöffenschulung zu, die nach dem Willen der SED in viertägigen Kursen unter Verantwortlichkeit des Innenministers und des Justizministers durchgeführt werden sollte ${ }^{79}$.

Die Bemühungen der SED im Hinblick auf die parteipolitische Zusammensetzung der Laienrichter waren erfolgreich. In jedem Land der SBZ brachten es die SED-Schöffen auf die absolute Mehrheit; im November 1947 gehörten von den für die 201-Prozesse gemeldeten 250 Schöffen 157 der SED, 45 der LDP, 39 der CDU und 9 keiner Partei an ${ }^{80}$. Die SED-Mehrheit läßt sich dabei nicht nur auf das Engagement der Einheitspartei, sondern auch darauf zurückführen, daß die anderen Parteien teilweise sogar auf die Benennung von Laienrichtern verzichteten, da sie nicht als bürgerliches ,Feigenblatt' dienen wollten. Hinzu kam, daß die Schöffen aus den bürgerlichen Parteien oft über die Art der Prozeßführung empört waren, aus den Strafkammern ausschieden und Platz für Nachrücker aus der SED machten ${ }^{81}$. Die Schöffenschulung war hingegen nicht durchgehend von Erfolg gekrönt. So stellte Generalstaatsanwalt Werner Fischl aus Halle Ende November 1947 der SED in diesem Zusammenhang ein denkbar schlechtes Zeugnis aus. Trotz Aufforderung des Landesvorstands durch Fischl, zur ersten Schöffenversammlung der drei Parteien einen guten Redner zu benennen, sei - mit erheblicher Verspätung - „so ein Würstchen geschickt [worden], das 7 Minuten lang Käse geredet hat. Die CDU hingegen schickte ihren besten Mann auf dem Gebiet der Justiz [...], der sehr geschickt die Frage der Schöffenschulung mit der Frage des Rechtsstaates verknüpfte und in Anspielungen gegen die SED und ihre Justizmoral Stellung nahm" 82 .

Die Richter und Schöffen erfüllten ihre Funktion als Vollstrecker des Polizeiund Parteiwillens zunächst jedoch mehr schlecht als recht. Insbesondere aus Thü-

77 Sitzungsprotokoll Landesblockausschuß, 1.9. 1947, in: Protokolle des Landesblockausschusses Brandenburg, S. 161.

78 Ergänzungsmaterial zum Rundschreiben betr. Entnazifizierung vom 10.9.1947 über die Anordnung des Befehls Nr. 201 durch die Schöffen, in: Rößler, Entnazifizierungspolitik, S. 173-181. Zum Problem der Kollektivschuld siehe unten, S 419-421.

79 Rundschreiben Nr. 21/4 des Zentralsekretariats, 10. 9. 1947, ebenda, S. 170.

80 Zahlen nach Wille, Entnazifizierung, S. $189 \mathrm{f}$. Etwas andere Zahlen enthält die nicht datierte Tabelle (S. 190), derzufolge von den 352 Schöffen 221 SED-, jeweils 51 CDU- und LDP-Mitglied und 29 parteilos waren.

81 Ebenda, S. 190.

82 Sonderbericht über eine Aussprache mit Fischl am 26. 11. 1947, BAB, DO1/7 Nr. 424, Bl. 94. 
ringen und Sachsen wurde berichtet, daß sich in den ersten Monaten nach Erlaß des Befehls zahlreiche für die Sonderstrafkammern vorgesehene Richter krank gemeldet hatten; in Thüringen sprach man in diesem Zusammenhang von der „201er-Krankheit“83. Zudem arbeiteten die Volksrichter nicht immer so, wie es sich die SED gedacht hatte: Vor dem SED-Ausschuß für Rechtsfragen beklagte sich jedenfalls ein sächsischer Vertreter über den zu großen „Formalismus“ mancher Absolventen bei den anlaufenden 201-Verfahren ${ }^{84}$. Auch die Schöffen spielten nicht die Rolle eines parteilichen Korrektivs gegenüber den vermeintlich konservativen Juristen. Im Gegenteil - Hilde Neumann kritisierte die „reaktionäre Einstellung" mancher selbst der SED angehörenden Schöffen, die überdies dem Gerücht aufsäßen, es handle „sich hier um ein politisches Strafrecht, um ein Gesinnungsstrafrecht " ${ }^{\text {"85. }}$. Melsheimer klagte Anfang Januar 1948 über Fälle, in denen "die Schöffen nicht so gestimmt haben, wie sie hätten stimmen sollen“, so daß harte Urteile abgemildert worden seien. Im Gegensatz zu Mielke, der sich dafür ausgesprochen hatte, in den 201-Verfahren in Abweichung von der Strafprozeßordnung bei der Abstimmung über das Urteil von der Zwei-Drittel-Mehrheit abzugehen, damit die Berufsrichter nicht „das Selbstbestimmungsrecht der Schöffen völlig" ausschalteten, wollte er aufgrund dieser Erfahrungen die bisherige Regelung jedoch beibehalten ${ }^{86}$. Im SED-Ausschuß für Rechtsfragen konnte Melsheimer sich vorerst nicht gegen Mielke durchsetzen ${ }^{87}$; erst Anfang November 1948 entschied Jakupow die Angelegenheit, der ganz im Sinne des DJV-Vizepräsidenten auf der uneingeschränkten Geltung der deutschen Strafprozeßordnung vor Gericht beharrte 88 .

Insgesamt fiel die Bilanz der SED-Juristen am 3./4. Januar 1948 negativ aus. Polak und andere hielten die Darlegungen über die Durchführung von Befehl Nr. 201 für "geradezu alarmierend“, da sie zeigten, daß man die sich selbst gestellte Bewährungsprobe nicht bestanden habe ${ }^{89}$. In diesen Chor der Kritiker einzustimmen und insgesamt zu konstatieren, der SED sei der Zugriff auf die Rechtsprechung nach Befehl Nr. 201 mißlungen ${ }^{90}$, geht indes zu weit. Vielmehr muß berücksichtigt werden, daß die Prozesse damals, wie Hans Nathan es ausdrückte, „erst im Anlaufen“ waren91. Eine SED-konforme Rechtsprechung war mit dem

83 Vgl. Weber, Rechtsstaat Thüringen?, S. 123.

84 Stenographische Niederschrift über die Tagung des Ausschusses für Rechtsfragen beim ZS, 3. 1./ 4. 1. 1948, SAPMO, DY 30 IV 2/1.01/70, Bl. 103, $111 \mathrm{f}$.

85 Ebenda, Bl. $132 \mathrm{f}$. (diese Stelle ausführlich zit. bei Meyer-Seitz, Verfolgung von NS-Straftaten, S. 198); Besprechung über den Befehl 201 und die damit verbundenen Aufgaben der Partei vom 30. 10. 1947, in: Rößler, Entnazifizierungspolitik, S. 193.

86 Stenographische Niederschrift über die Tagung des Ausschusses für Rechtsfragen beim ZS, 3. 1./ 4. 1. 1948, SAPMO, DY 30 IV 2/1.01/70, Bl. 125, 110; Protokoll der Tagung über den Befehl 201 am 22. 12. 1947, in: Rößler, Entnazifizierungspolitik, S. $241 \mathrm{f}$. (dort auch das Zitat).

$87 \mathrm{Vgl}$. die Stellungnahme des rechtspolitischen Beirats zur Durchführung der Verfahren nach Befehl 201, Punkt 6, SAPMO, DY 30 IV 2/2.1/162, Anlage Nr. 3.

88 Vermerk Gertichs über Besprechung bei Rechtsabteilung und Abteilung Inneres der SMAD, 2. 11. 1948, BAB, DO1/7 Nr. 436, Bl. 78. Vgl. insgesamt Meyer-Seitz, Verfolgung von NS-Straftaten, S. $251 \mathrm{f}$.

89 Stenographische Niederschrift über die Tagung des Ausschusses für Rechtsfragen beim ZS, 3. 1./ 4. 1. 1948, SAPMO, DY 30 IV 2/1.01/70, Bl. 135.

90 So Meyer-Seitz, Verfolgung von NS-Straftaten, passim, insbesondere S. $346 \mathrm{f}$.

91 Stenographische Niederschrift über die Tagung des Ausschusses für Rechtsfragen beim ZS, 3. 1./ 4. 1. 1948, SAPMO, DY 30 IV 2/1.01/70, Bl. 142. 
zur Verfügung stehenden Justizapparat nicht über Nacht zu erreichen. Die SED setzte sich jedoch, wie bereits im Zusammenhang mit der Besetzung der 201Strafkammern gezeigt, 1948/49 in mehrfacher Hinsicht durch, so daß die Bedeutung von Befehl Nr. 201 für die Transformation des Justizwesens im Sinne der Einheitspartei gar nicht hoch genug eingeschätzt werden kann.

Während die SED vor allem über den Personaleinsatz die Prozesse zu steuern suchte, wollte die DVdI insbesondere über die von den Untersuchungsorganen verfaßten Anklageschriften deren Ausgang präjudizieren. Als Untersuchungsorgan fungierte eine seit dem Spätsommer 1945 zunächst in Sachsen, ab 1946 auch in den anderen Ländern aufgebaute Abteilung der Kriminalpolizei, die seit Januar 1947 als K 5 bezeichnet wurde. Von Anfang an als politische Polizei konzipiert, stand an ihrer Spitze das Referat K 5 in der Abteilung Kriminalpolizei der DVdI; in den Landeskriminalämtern wurden entsprechende Dezernate, in den Kriminalaußenstellen entsprechende Kommissariate ins Leben gerufen. Unter enger Anleitung des MWD/MGB aufgebaut, unterstützten sie die Besatzungsmacht in ihrem Kampf gegen vermeintliche Saboteure und politische Gegner ${ }^{92}$. Als die K 5 mit den Aufgaben des Untersuchungsorgans in den 201-Verfahren betraut wurde, bedeutete dies eine enorme Ausweitung ihres Tätigkeitsfeldes; infolgedessen erfuhr sie nach den Angaben Mielkes im Herbst 1947 in allen Ländern eine Verstärkung um 875 aus der Schutzpolizei stammenden Kräfte; im Juni 1949 verfügte sie über knapp 1600 Mitarbeiter ${ }^{93}$. Das Aufgabenspektrum der K 5 hatte somit zwar einen neuen Schwerpunkt erhalten; mit ihrer Verstärkung ging jedoch auch eine Diversifizierung der Aufgaben einher. Ab Januar 1948 war die K 5 in vier Sektionen untergliedert: Die erste befaßte sich mit Verstößen gegen SMAD-Befehle, die zweite mit Verstößen gegen Kontrollratsanordnungen (unter anderem KRD 38, bzw. Befehl Nr. 201), die dritte mit gegen den demokratischen Neuaufbau gerichteten Sabotageakten und die vierte mit sonstigen antidemokratischen Aktivitäten ${ }^{94}$.

Zwar trat die K 5 mit dem Anspruch auf, die Gerichtsverfahren nach Befehl Nr. $201 \mathrm{zu}$ dominieren; die neuen, in aller Regel juristisch völlig unbedarften Kräfte, bei deren Einstellung hauptsächlich auf politische Zuverlässigkeit geachtet worden war'5, erwiesen sich jedoch oft als unfähig, die Ermittlungen korrekt durchzuführen und Anklageschriften zu verfassen, die allen inhaltlichen und formalen Erfordernissen entsprachen und somit vor Gericht Bestand hatten. So kritisierte der thüringische Generalstaatsanwalt Kuschnitzky, daß die Vernehmungen durch die K 5 ,vielfach zur Klärung des Sachverhaltes nicht ausreichend“ seien, der Beschuldigte oft nicht gehört werde, Aussagen Dritter als glaubhaft bezeichnet würden, ohne diese zu vernehmen, und Entlastungszeugen in den Vorgängen kaum vorkämen ${ }^{96}$. Um derartigen Mißständen abzuhelfen, sollten ab dem

92 Vgl. insgesamt Naimark, Russians in Germany, S. 360f.; Gieseke, Ministerium für Staatssicherheit, S. 373 f.; Nollau, Amt, S. 95 f.

93 Bericht Mielkes vom 30. 10. 1947, in: Rößler, Entnazifizierungspolitik, S. 208; Gieseke, Ministerium für Staatssicherheit, S. 374.

94 Vgl. Naimark, Russians in Germany, S. 362; Pohl, Justiz in Brandenburg (Manuskript), S. 117.

95 So der zutreffende Vorwurf Fischls in der Länderkonferenz vom 14./15. 11. 1947, BAB, DP1 VA Nr. 6332.

96 Ebenda. Für Thüringen vgl. Weber, Justiz und Diktatur, S. 111. 
3. November 1947 in der Höheren Polizeischule in Berlin sechs dreitägige Lehrgänge für K 5-Mitglieder durchgeführt werden, in denen diese über die politische Bedeutung von Befehl Nr. 201, die entstehenden administrativen Aufgaben der Kriminalpolizei, die strafrechtlichen Bestimmungen und Auslegungen sowie „die Beibringung ,sachlicher Beweise “" in diesen Verfahren zu unterrichten waren"7. Schulungen der Kriminalpolizisten fanden außerdem, vor Ort' statt: Am 4./5. Dezember 1947 wurden beispielsweise unter Anleitung des aufsichtsführenden Staatsanwalts Julie Ganske 40 Kriminalpolizisten am Landgericht Dessau mit den juristischen Feinheiten der 201-Verfahren vertraut gemacht ${ }^{98}$.

Mit derartigen Schulungen konnten die Schwächen in der Vernehmungstaktik, in der Beweisführung und in der Anklageschrift jedoch nicht behoben werden ${ }^{99}$. So wurde im Februar 1948 kritisiert, daß die Beweismittel vielfach zu schwach und fehlerhaft zusammengetragen, die Anklageschriften unvollständig und die Angeklagten unkorrekt in die Kategorien des Anhangs A von Direktive 38 eingestuft seien. Die Untersuchungsorgane verstießen des weiteren gegen die Ausführungsbestimmung Nr. 3, insbesondere durch die Inhaftierung von Personen, bei denen kein genügender $A n l a ß$ vorhanden war, und indem sie es versäumten, ihren Haftbefehl vom Staatsanwalt bestätigen zu lassen oder diesen zu spät von einem Untersuchungsvorgang in Kenntnis setzten ${ }^{100}$. Das ohnehin nur schwach ausgeprägte Kontrollrecht des Staatsanwalts wurde auf diese Weise weiter ausgehöhlt. Das in einem weitgehend rechtsfreien Raum stattfindende Untersuchungsverfahren bedingte schließlich äußerst rüde Vernehmungsmethoden. Der Beschuldigte besaß weder die Möglichkeit einer Haftbeschwerde, noch konnte er im Untersuchungsverfahren einen Anwalt hinzuziehen ${ }^{101}$; nach einer Anweisung der Abteilung Kriminalpolizei der DVdI war ihm sogar - außer in Sterbefällen - „seelsorgerische Fürsorge zu versagen" ${ }^{102}$. Die Vorgehensweise der Vernehmer erinnerte daher teilweise, wie das Untersuchungsorgan aus Mecklenburg am 22. Dezember berichtete, „an die Methoden der Gestapo“. Mielke empfahl zwar, die Verhaftungen einzuschränken und damit sicherzustellen, „daß in Zukunft keine Gestapo-Methoden mehr angewandt werden", machte aber unmißverständlich klar: „Das heißt aber nicht, daß man nun die Untersuchungen ohne die notwendige Härte führt. " ${ }^{103}$ Vor diesem Hintergrund verbreitete die K 5 nicht nur in den ersten Monaten nach Erlaß von Befehl Nr. $201^{104}$ Angst und Schrecken. Dem gab Bischof Dibelius in seinem Pfingsthirtenbrief vom 1. Juni 1949 allgemeinen Aus-

97 Bericht Mielkes vom 30. 10. 1947, in: Rößler, Entnazifizierungspolitik, S. 209; Vermerk Abteilung K, 1. 11. 1947, BAB, DO1/7 Nr. 421, Bl. 55.

98 Ganske an DJV, 8. 12. 1947, BAB, DP1 VA Nr. 6204, Bl. 47.

99 Vgl. Vermerk für die Chef-Besprechung, 10. 2. 1948, BAB, DO1/7 Nr. 421, Bl. 108.

100 Für diese Vorwürfe siehe: DVdI, Referat K 5, an Chefs der LBDVP, Betr.: Weitere Durchführung des Befehls Nr. 201, 14. 6. 1948, BAB, DP1 VA Nr. 6200, Bl. $21 \mathrm{f}$.

101 So Jakupow bei der Länderkonferenz vom 14./15. 11. 1947, BAB, DP1 VA Nr. 6223; vgl. auch den Hinweis Mielkes in einer Besprechung vom November 1947, BAB, DO1/7 Nr. 421, Bl. 65.

102 DVdI, Abteilung K, an Leiter der Landeskriminalämter, 20. 1. 1948, ebenda, Bl. 103.

103 Protokoll der Tagung über den Befehl 201 am 22. 12. 1947, in: Rößler, Entnazifizierungspolitik, S. 226, 239. Von "Gestapomethoden" der K 5 war auch in Brandenburg die Rede: vgl. Pohl, Justiz in Brandenburg (Manuskript), S. $143 \mathrm{f}$.

104 So mißverständlich Meyer-Seitz, Verfolgung von NS-Straftaten, S. 241. 
druck, in dem es hieß: „In der Abteilung K 5 der sogenannten Volkspolizei ist die Gestapo unseligen Angedenkens wieder erstanden. ${ }^{105}$

Das mangelhafte und rechtsstaatswidrige Vorgehen der Untersuchungsorgane hatte vor der Justiz oft keinen Bestand. Die aufsichtsführenden Staatsanwälte ließen nicht jede Anklage passieren. Bis zum 25. Dezember 1948 bestätigten sie von den insgesamt erhaltenen 14539 Anklagen 10190 und gaben 2418 entweder zur Vervollständigung an die Untersuchungsorgane zurück oder lehnten sie endgültig ab (1931 Fälle waren von den Staatsanwaltschaften noch nicht erledigt) ${ }^{106}$. Dabei bestanden von Land zu Land jedoch erhebliche Unterschiede. Während etwa in Brandenburg bis Jahresende 1947 die Staatsanwaltschaft nur acht Anklageschriften zurückwies, wurden allein in Dessau im September 1948 von 24 Anklageschriften 18 zurückgereicht ${ }^{107}$. Allem Anschein nach waren es weniger die weitgehend regimetreuen Staatsanwälte als die Gerichte, die vorerst nicht so funktionierten, wie sich die Untersuchungsorgane dies vorstellten. Ein Indiz dafür ist die ständige Kritik der DVdI an den zu milden Urteilen, den Freisprüchen und Verfahrenseinstellungen: Derartige gerichtliche Entscheidungen, so Mielke am 10. Mai 1948, entsprächen nicht den Untersuchungsergebnissen ${ }^{108}$. Ein wesentlicher Grund für diese Diskrepanz bestand darin, daß die Vernehmungen im Gerichtssaal ein völlig anderes Bild als die polizeiliche Untersuchung ergaben. „Oft", so der thüringische Generalstaatsanwalt im Juni 1948, „würden polizeiliche Aussagen mit der Behauptung widerrufen, die Angaben seien unter Druck gemacht worden oder der vernehmende Polizeibeamte habe trotz Einwendungen des Vernommenen Protokolländerungen abgelehnt. ${ }^{109}$ Richter gingen mitunter sogar so weit wie Landgerichtspräsident Leopold Friedensburg (CDU) aus Meiningen, der bei der Eröffnung der gerichtlichen Hauptverhandlung die Angeklagten bat, „sich nunmehr frei und ohne Hemmungen zu äußern, denn sie ständen nicht mehr vor den Untersuchungsorganen"110. Daß unter diesen Bedingungen Anklagen zusammenbrachen, die auf fragwürdigen Beweisen und erzwungenen Geständnissen beruhten, ist nicht weiter verwunderlich. Die DVdI versuchte dem entgegenzusteuern, indem sie die Landespolizeibehörden auf die von seiten der Justiz genannten Mängel hinwies und vor allem die Einhaltung der Ausführungsbestimmungen von Befehl Nr. 201 anmahnte ${ }^{111}$. Es ist indes nicht anzunehmen, daß sich dadurch die Praktiken der Untersuchungsbehörden wesentlich änderten. Was sich demgenüber sehr wohl auf die Dauer änderte, war die Besetzung der Strafkammern. So wurden etwa in Thüringen Friedensburg und zwei andere Richter "wegen Versagens in 201-Verfahren“ entlassen. Auch Kuschnitzky, der immer

105 Zit. nach Kirchliches Jahrbuch 1949, S. 233.

106 DJV an Chef der SMAD-Rechtsabteilung, 12. 1. 1949, Bericht über die Geschäftsentwicklung der Justizbehörden im 2. Halbjahr 1948, BAB, DP1 VA Nr. 6469.

107 Pohl, Justiz in Brandenburg (Manuskript), S. 148; Zusammenstellung einiger Schwierigkeiten der Untersuchungsorgane in Zusammenarbeit mit SMA- und Justizdienststellen, 1.10. 1948, BAB, DO1/7 Nr. 424, Bl. 274.

108 Vermerk Mielkes, 10.5. 1948, DO1/7 Nr. 436, Bl. 35. Der Vermerk ging sowohl an die Landeskriminalämter als auch an die SMAD-Innenverwaltung und Rechtsabteilung.

109 Protokoll der Juristenkonferenz vom 11./12. 6. 1948, BAB, DP1 VA Nr. 264, Bl. 18.

110 DVdI, Referat K 5, an SMAD-Rechtsabteilung, 2. 7. 1948, BAB, DO1/7 Nr. 436, Bl. 66.

111 DVdI, Referat K 5, an Chefs der LBDVP, Betr.: Weitere Durchführung des Befehls Nr. 201, 14. 6. 1948, BAB, DP1 VA Nr. 6200, Bl. $21 \mathrm{f}$. 
wieder auf die Einhaltung von rechtsstaatlichen Verfahrensgrundsätzen gedrängt hatte, mußte im September 1948 aus dem Justizdienst ausscheiden ${ }^{112}$.

Während dafür die sowjetischen Militärverwaltungen der Länder zuständig waren, beschränkte sich die DJV im wesentlichen darauf, die Rechtsprechung zunächst über Artikel in der Neuen Justiz ${ }^{113}$ und Rundverfügungen zu steuern. Melsheimer hatte auf der Länderkonferenz vom 14./15. November 1947 angekündigt, bei Zweifelsfragen in Zusammenarbeit mit der SMAD-Rechtsabteilung entsprechende „Instruktionen" zu erlassen"14. Eine entsprechende ausführliche Rundverfügung war seit Februar 1948 in Arbeit; der endgültige, von Melsheimer am 25. März abgezeichnete Entwurf ${ }^{115}$ traf auf Bedenken der SMAD-Rechtsabteilung, und Jakupow beauftragte Weiß, die Rundverfügung zu überarbeiten und erheblich zu kürzen ${ }^{116}$. Nach den Ausführungen Melsheimers auf der Justizkonferenz vom Juni 1948 sah Karlshorst anscheinend nicht mehr die Notwendigkeit einer solchen Rundverfügung, so daß der Entwurf unbestätigt blieb ${ }^{117}$.

Die DJV mußte sich daher auf Einzelanweisungen beschränken. Auch dabei war sie oft nicht Urheberin, sondern lediglich Übermittlerin von Anweisungen anderer Instanzen. Dies wird unter anderem an der Frage deutlich, ob die 201Strafkammern auf Internierung als Strafe erkennen konnten. Kontrollratsdirektive Nr. 38 sah diese Strafart für Hauptschuldige und Belastete ausdrücklich vor, und Weiß hatte dies auf der Länderkonferenz vom 14./15. November bekräftigt, jedoch dabei auf den Sicherungscharakter der Internierungsstrafe aufmerksam gemacht, die somit nur dann anzuwenden sei, wenn es gelte, "die Gesellschaft vor einem politisch gefährlichen Menschen zu sichern" ${ }^{118}$. Erst nachdem aufgrund der Kritik der Innenverwaltung die Unzulässigkeit dieser Art der Bestrafung festgestellt worden war, wies die DJV am 9. Februar 1948 die Justizministerien in einem entsprechenden Sinne an ${ }^{119}$. Auch bei der ab Sommer 1948 aufgrund der Entlassung von über 27000 Personen aus den Speziallagern ${ }^{120}$ aktuell gewordenen Frage, ob Internierung in diesen Lagern auf eine Haftstrafe anzurechnen sei, änderte sich die Auffassung der DJV. Ging Schiffer am 30. Juli noch davon aus, daß diese Frage zu bejahen sei, antwortete die DJV am 5. November auf eine entsprechende An-

112 Vgl. Weber, Rechtsstaat Thüringen?, S. $123 \mathrm{f}$. Daraus geht auch hervor, daß mehr Richter abberufen wurden, als von Meyer-Seitz, Verfolgung von NS-Straftaten, S. 194 f., aufgeführt.

113 Vgl. Benjamin, Zum SMAD-Befehl Nr. 201; Guski, Rechtsfragen zum Befehl Nr. 201; Winkelmann, Aufgaben der Staatsanwälte und Gerichte. Diese Artikel waren jedoch „eher Erläuterung als Anweisung": siehe Meyer-Seitz, Verfolgung von NS-Straftaten, S. 202.

114 Protokoll der Länderkonferenz am 14./15. 11. 1947, BAB, DP1 VA Nr. 6332.

115 Der Entwurf der Rundverfügung datiert vom April 1948, BAB,DP1 VA Nr. 6200, Bl. 52-61.

116 Vermerk Weiß über Unterredung mit Jakupow am 4. 5. 1948, BAB, DP1 VA Nr. 7132, Bl. 97.

117 Vgl. zur Entstehung und Ablehnung der DJV-Rundverfügung auch Meyer-Seitz, Verfolgung von NS-Straftaten, S. 201-205. Für seine Behauptung, diese sei aufgrund des Einspruchs der SED abgelehnt worden, fehlt jeder Hinweis ebenso wie für die These, die Rundverfügung habe einen Konflikt zwischen den zuständigen Referenten, Hirschfeld und Weiß, hervorgerufen.

118 Protokoll über die Konferenz mit den Vertretern der Justizministerien der Landesregierungen am 14./15. 11. 1947, BAB, DP1 VA Nr. 6332.

119 Die DVdI hatte kritisiert, daß Internierung von einigen Kammern als „eine neben der Gefängnisstrafe stehende mildere Strafart" verhängt werde: Mielke an DJV, 21.2. 1948, BAB, DO1/7 Nr. 424, Bl. 183. Als Beispiel einer entsprechenden Anweisung DJV an Justizministerium SachsenAnhalt, 20. 2. 1948, BAB, DP1 VA Nr. 6204, Bl. 98.

120 Eine sowjetische Kommission schlug 27749 Personen zur Entlassung vor: siehe Petrov, Apparate des NKVD/MVD, S. $150 \mathrm{f}$. 
frage des thüringischen Justizministeriums: „Vorbeugende Haft - verhängt von einer Besatzungsmacht gegen gefährliche oder möglicherweise gefährliche Personen - kann von deutschen Gerichten nicht auf eine Entlassung aus dieser Haft verhängte Strafe angerechnet werden. " $121 \mathrm{Zwar}$ ist bei dieser auf eine Besprechung bei Melsheimer zurückgehende Entscheidung ${ }^{122}$ weder eine vorherige Anweisung der SMAD-Rechtsabteilung noch eine Absprache mit der DVdI nachweisbar; jedoch hatte sich der Leiter der SMA-Rechtsabteilung in Thüringen, Schur, bereits gegen die Anrechnung von Internierungshaft ausgesprochen ${ }^{123}$. Diese Antwort war anscheinend nicht mit der SMAD-Rechtsabteilung in Karlshorst abgestimmt; die DJV hatte sich aber vorsorglich die Auffassung eines anderen führenden sowjetischen Justizvertreters zu eigen gemacht. Die noch von Schiffer vertretene Meinung entsprach der in der britischen Zone üblichen Praxis; das von der DJV im November 1948 unter einem neuen Präsidenten angeordnete und mit Rundverfügung vom 30 . September 1949 bekräftigte Anrechnungsverbot ${ }^{124}$ widersprach einem grundsätzlichen Gerechtigkeitsgebot und zeigt, daß sich nun auch die Zentrale Justizverwaltung von rechtsstaatlichen Grundsätzen mehr und mehr entfernte ${ }^{125}$.

Sehr viel wichtiger für die Staatsanwaltschaften und Gerichte war das Problem, inwieweit Befehl Nr. 201 den Strafkammern die Möglichkeit einräumte, Angeklagte allein aufgrund ihrer Zugehörigkeit zu einer NS-Organisation zu verurteilen. Diese Möglichkeit war aufgrund Direktive Nr. 38 gegeben bei sogenannten Hauptschuldigen, also bei denen, die sich in führender Stellung in der NSDAP, in einer ihrer Gliederungen oder in der Regierung des Reichs, der Länder oder der besetzten Gebiete engagiert hatten. In dieser Auffassung stimmten sowohl die Kommunistin Benjamin als auch der Sozialdemokrat Guski überein ${ }^{126}$; strittig war, ob allein die Zugehörigkeit zu einer in Anhang A I aufgeführten Gruppierung die Strafbarkeit begründete, da dieser auch Personengruppen umfaßte, die keine leitenden Stellungen eingenommen hatten. Da die meisten Regierungsbeamten und höheren NS-Funktionäre jedoch entweder bereits von der sowjetischen Besatzungsmacht festgenommen worden waren oder sich in die Westzonen abgesetzt hatten, fielen kaum Angeklagte in diese Kategorie ${ }^{127}$. Die einfachen SS-, SDund Gestapo-Mitglieder konnten nach Direktive Nr. 38 nur dann aufgrund ihrer Organisationszugehörigkeit verurteilt werden, wenn sie sich dort aktiv betätigt hatten ${ }^{128}$. Diese Ausnahmeregel wurde jedoch in der SBZ von Anfang an nicht beachtet. Die SED legte in ihrem Schulungsmaterial für die Schöffen vom September 1947 ausführlich dar, daß bei den Mitgliedern von in Nürnberg für verbrecherisch erklärten Organisationen kein individueller Schuldnachweis für eine Verurteilung

121 Chef der DJV an SMAD-Rechtsabteilung, 30. 7. 1948, BAB, DP1 VA Nr. 7132, Bl. 116f.; Land

Thüringen, Ministerium für Justiz, an DJV, 22. 9. 1949, BAB, DP1 VA Nr. 6201, B1. 1; DJV an

Landesregierung Thüringen, 5. 11. 1948, ebenda, Bl. 6.

122 Dies geht aus einer Notiz Reislers vom 14.6. 1949, ebenda, Bl. 14, hervor.

${ }^{123}$ Land Thüringen, Ministerium für Justiz, an DJV, 22. 9. 1949, ebenda, Bl. 1.

124 Chef der DJV an Landesregierungen/Justizministerium, 30.9. 1949, ebenda, Bl. 21.

125 Vgl. auch Meyer-Seitz, Verfolgung von NS-Straftaten, S. 323-325, mit einer ähnlichen Bewertung.

126 Siehe Benjamin, Zum SMAD-Befehl Nr. 201, S. 151; Guski, Rechtsfragen zum Befehl Nr. 201,

S. 173.

127 Vgl. insgesamt dazu Meyer-Seitz, Verfolgung von NS-Straftaten, S. 264-267.

128 Vgl. ebenda, S. 274. 
erforderlich sei, und auch Mielke betonte ohne jede Differenzierung, „daß eine allgemeine Schuld genügen müsse und daß für den Prozeß ein Nachweis der persönlichen Schuld im Einzelfall zu fordern, falsch sei“"129. Demgegenüber war die DJV in ihrem Rundverfügungsentwurf vom April 1948 weitaus vorsichtiger. Sie nahm zwar auch keinen Bezug auf die Einschränkungen des Nürnberger Tribunals, legte aber dar, daß nur bei nachgewiesener Betätigung in führender Stellung „Einstufung und Verurteilung des Angeklagten nicht an den Nachweis verbrecherischer Einzelhandlungen gebunden ist". Die anderen Einstufungen seien jedoch vom „beweisbaren Sachverhalt“ abhängig ${ }^{130}$. Auf der Justizkonferenz im Juni 1948 gab Melsheimer indes deutlich zu verstehen, daß der wesentliche Sinn von Befehl Nr. 201 darin bestehe, „die verbrecherische Gesinnung des Täters innerhalb des Verbrecherkollektivs der Nazibande zu erfassen und den Täter wegen seiner Mitwirkung im Rahmen des an Deutschland und der Welt begangenen Massenverbrechens zu bestrafen "131. Nun änderte sich auch die Haltung der DJV in dieser Frage. Bei einer Unterredung mit zwei Angehörigen des Referats K 5 teilten Melsheimer und Guski die Auffassung, daß SS-Angehörige bestraft werden konnten, wenn diese einem Verband angehört hatten, der an verbrecherischen $\mathrm{Ak}$ tionen beteiligt gewesen war. „Eine ,persönliche Schuld““, so hielt der Leiter der Rechtsstelle im K 5-Referat fest, „braucht in diesem Falle nicht nachgewiesen werden, es genügt hier die ,Kollektiv-Schuld"“"132 Zwar stimmte die DJV in der Sache damit überein; nur den Begriff wollten ihre Vertreter auch weiterhin vermeiden, da er "Mißverständnisse hervorrufen“ könne133. Am 17. Juli forderte Melsheimer, daß zur bloßen Zugehörigkeit zur SS „schon etwas Positives hinzukommen [müsse], z.B. daß durch dauerndes Tragen der SS-Uniform ein offenes Bekenntnis zur nationalsozialistischen Gewaltherrschaft abgelegt wurde, oder daß die betreffende Formation, der das Mitglied angehört, bei besonderen Einsätzen, z. B. in Polen als KZ-Wachmannschaft oder dergleichen teilgenommen hat". Eine individuelle strafbare Handlung müsse jedoch nicht nachgewiesen werden ${ }^{134}$. Daher konnte die DJV auch dem Muster einer Anklageschrift „gegen einen sonst nicht weiter belasteten SS-Mann“ zustimmen, das die DVdI im April den Untersuchungsorganen zur Verfügung gestellt hatte, und beschränkte sich auf einige wenige kosmetische Korrekturvorschläge ${ }^{135}$. Die DJV bejahte somit auch die

${ }^{129}$ Ergänzungsmaterial zum Rundschreiben betr. Entnazifizierung vom 10.9. 1947, in: Rößler, Entnazifizierungspolitik, S. $177 \mathrm{f}$. Mielke äußerte sich vor der Justizkonferenz am 11./12.6. 1948, BAB, DP1 VA Nr. 264, Bl. $15 \mathrm{f}$.

130 Rundverfügungsentwurf, BAB, DP1 VA Nr. 6200, Bl. 54.

131 Referat Melsheimers in: Die Juristenkonferenzen, S. 129.

132 Vermerk über Unterredung mit Melsheimer und Guski am 21.6. 1948, 22. 6. 1948, BAB, DO1/7 Nr. 424, Bl. 235.

${ }_{133}$ DJV an DVdI, 11. 8. 1948, ebenda, Bl. 262.

134 Chef der DJV an DVdI, 17. 7. 1948, ebenda, Bl. 251. Damit reagierte Melsheimer auf das auch der DJV zugesandte Schreiben Mielkes an die Chefs der LBDVP vom 14.6. 1948, BAB, DP1 VA Nr. 6200, Bl. 22, in dem es hieß: „Eine allgemeine Schuld dürfte bei dem Charakter dieser Organisation mit wenigen Ausnahmen immer nachzuweisen sein, denn die Tatbestandsmerkmale der Direktive 38 sind fast immer bei SS-Leuten gegeben."

135 Muster einer Anklageschrift, ebenda, Bl. 26; dazu DJV an DVdI, 11.8. 1948, DO1/7 Nr. 424, Bl. 262. Die Korrekturvorschläge müssen nicht, wie Meyer-Seitz, Verfolgung von NS-Straftaten, S. $276 \mathrm{f}$., behauptet, auf starke Spannungen zwischen DVdI und DJV hindeuten; wahrscheinlicher ist, daß die DJV ein Scheitern der Untersuchungsbehörden mit ihrer Anklageschrift an den Staatsanwälten oder Gerichten verhindern wollte. 
Bestrafung nach Kontrollratsdirektive allein aufgrund eines Organisationsdelikts. Aufgrund dieser Vorgabe verurteilten die Gerichte bis zum Oktober 1949 insgesamt 3037 Personen, von denen 1055 der SS, jeweils 41 der Gestapo oder dem SD angehört, 737 sich als politische Leiter der NSDAP und 1163 als SA-Führer betätigt hatten. Bei einer Gesamtzahl von 7470 zum damaligen Zeitpunkt in den 201-Prozessen Verurteilten handelte es sich somit um 40 Prozent, bei denen eine Kollektivschuld geltend gemacht wurde ${ }^{136}$.

Während bei der Verurteilungsgrundlage offensichtlich die Steuerungsmechanismen funktionierten, galt dies nicht durchgehend für das verhängte Strafmaß. Trotz aller Ermahnungen zu harten Urteilen blieb der Anteil der leichten Freiheitsstrafen relativ konstant. Nach einer Übersicht über die Anzahl der Verurteilten vom Inkrafttreten des Befehls Nr. 201 bis zum 30. Juni 1948 waren 564 Personen zu Gefängnisstrafen oder Internierung unter einem Jahr verurteilt worden. Das entsprach bei insgesamt 3144 Verurteilten einem Anteil von 17,9 Prozent. $\mathrm{Zu}$ ein bis drei Jahren Gefängnis oder Internierung waren 1171 Personen (also 37,2 Prozent) verurteilt worden ${ }^{137}$. Vor allem letztere Zahl blieb ziemlich konstant: In einem Vermerk vom 26. Oktober 1949 notierte der Berichterstatter, daß auch von den damals 7470 Verurteilten 40 Prozent Freiheitsstrafen zwischen ein und drei Jahren erhalten hatte ${ }^{138}$. Die Freiheitsstrafen unter einem Jahr unterlagen hingegen einer signifikanten Schwankung. Aufgrund der mit SMAD-Befehl Nr. 43 ausgesprochenen Amnestie vom 18. März 1948, die gegen den Willen der führenden SED-Mitglieder in der DJV auch die nach Befehl Nr. 201 Verurteilten betraf ${ }^{139}$, waren nicht nur alle zu Freiheitstrafen von bis zu einem Jahr verurteilten Personen zu entlassen, sondern auch die Verfahren einzustellen, die vor Erlaß des Befehls eingeleitet worden waren, bei denen aber eine Strafe unter einem Jahr erwartet wurde ${ }^{140}$. Dies führte in den Monaten April bis Mai zu einer erheblichen Absenkung der Quote der Verurteilungen unter einem Jahr von 16,8 Prozent im Monat vor der Amnestie (26. Februar bis 25. März) auf 10 Prozent. Denn nach der Verkündung der Amnestie tendierten die Gerichte dazu, 13 oder 14 Monate Freiheitsstrafe zu verhängen, wo sie zuvor, um eine völlige Freistellung von der Strafe zu vermeiden, auf Strafen knapp unter einem Jahr erkannt hatten. Ab dem Juni 1948 pendelte sich dieser Prozentsatz wieder bei 15 ein $^{141}$. Das Verhältnis von Freisprüchen und Verfahrenseinstellungen auf der einen und Verurteilungen auf der anderen Seite veränderte sich sogar leicht zugunsten der ersteren: So stieg die Zahl der Verurteilten von 3144 am 30. Juni 1948 auf 5147 am 25. Dezember 1948 und 8321 Ende 1950 weniger stark an als die Zahl der Freisprüche und Verfahrenseinstellungen, die sich im gleichen Zeitraum von 450 und 1050 auf 826 und 2042

136 Vermerk Reislers, Betr.: Ergebnisse der bisherigen 201-Rechtsprechung, 26. 10. 1949, BAB, DP1 VA Nr. 6229; vgl. Meyer-Seitz, Verfolgung von NS-Straftaten, S. $277 \mathrm{f}$. Dort auch Beispiele aus der Verurteilungspraxis.

137 Übersicht in: BAB, DP1 VA Nr. 580, Bl. 249.

138 Vermerk Reislers, Betr.: Ergebnisse der bisherigen 201-Rechtsprechung, 26. 10. 1949, BAB, DP1 VA Nr. 6229.

139 Vgl. dazu Melsheimer, Benjamin, Weiß an Fechner, 31. 3. 1948, BAB, DP1 VA Nr. 6596, Bl. 62-64;

Meyer-Seitz, Verfolgung von NS-Straftaten, 214-219.

140 Abgedruckt in: Neues Deutschland, 18. 3. 1948.

141 Vgl. Meyer-Seitz, Verfolgung von NS-Straftaten, S. 227. 
und auf 1500 und 3100 erhöhten. Urteile mit Höchststrafen wurden hingegen sehr viel häufiger ausgesprochen: Während bis zum 30. Juni 1948 lediglich sechs $\mathrm{Mal}$ die Todestrafe, fünf Mal lebenslänglich und $18 \mathrm{Mal} \mathrm{Zuchthaus} \mathrm{zwischen} 10$ und 15 Jahren verhängt worden war, hatten sich diese Zahlen im Oktober 1949 auf 23142, 36 und 87 gesteigert. Dies bedeutete unter Zugrundelegung der Gesamtzahl aller Verurteilten auch eine erhebliche prozentuale Erhöhung ${ }^{143}$. Insgesamt waren die Gerichte trotz aller Einwirkungsversuche insgesamt bei vergleichsweise milden Strafen geblieben und hatten sich auch nicht gescheut, einen erheblichen Anteil der Angeklagten straffrei ausgehen zu lassen; es hat jedoch den Anschein, als ob die Hemmschwelle bei der Verhängung der Höchststrafen herabgesetzt wurde.

Während die Entnazifizierungskommissionen aufgrund von SMAD-Befehl Nr. 35 vom 26. Februar 1948 zum 10. März ihre Tätigkeit einstellten ${ }^{144}$, war damals ein Ende der 201-Verfahren noch nicht abzusehen. Im Herbst 1948 drängte die DJV zwar wiederholt auf deren möglichst rasche Beendigung ${ }^{145}$. Nachdem die Anzahl der Prozesse im Herbst 1948 tatsächlich auch etwas zurückgegangen war, stieg sie jedoch wieder an, nachdem DJV und DVdI mit einer gemeinsamen Rundverfügung bekanntgegeben hatten, daß die Wirkung der Amnestie in den 201-Verfahren sich lediglich auf die Haft- und Geldstrafen, nicht aber auf die verhängten Sühnemaßnahmen - wie z.B. die Vermögenseinziehung - beziehe ${ }^{146}$. Während in der DVdI diese Entwicklung eher mit Gleichmut betrachtet wurde ${ }^{147}$, plädierte der in der DJV bzw. im MdJ zuständige Referent, Erwin Reisler, im Oktober 1949 dafür, durch eine Ausweitung der Amnestie - die nun bei Haftstrafen bis zu zwei Jahren greifen sollte - die Verfahren ,in einer absehbaren Zeit (geplant ist möglichst noch bis Jahresende) in der Mehrzahl abzuschließen“. Denn zu Recht sah er in der durch Befehl Nr. 201 begründeten Verpflichtung, auch weniger schwere NS-Verbrechen zu sühnen, eine Hypothek für die gerade erst gegründete DDR. Nun kam es Reisler zufolge darauf an, die Integration der ,kleinen Nazis' in den neuen Staat nicht durch die Fortführung der 201-Prozesse zu gefährden, zumal es sich bei den Tätern „leider zum größten Teil um Angehörige der Arbeiterklasse handelt“, die man aber keineswegs als Gegner der „antifaschistisch-demokrati-

142 Nach Werkentin, Souverän, S. 181, sprachen bis 1949 deutsche Gerichte in der SBZ 43 Todesurteile wegen NS-Verbrechen aus, von denen 25 nachweislich vollstreckt wurden; dazu zählen jedoch auch $10 \mathrm{KRG}-10$-Urteile von 1946 und 1947.

${ }^{143}$ Für die Zahlen siehe: Übersicht über die nach Direktive 38 in Durchführung des Befehls Nr. 201 verurteilten Personen vom Inkrafttreten des Befehls bis 30.6. 1948, BAB, DP1 VA Nr. 580, Bl. 249; Bericht über die Geschäftsentwicklung der Justizbehörden im 2. Halbjahr 1948, BAB, DP1 VA Nr. 6469; Vermerk Reislers, Betr.: Ergebnisse der bisherigen 201-Rechtsprechung, 26. 10. 1949, BAB, DP1 VA Nr. 6229; Meyer-Seitz, Verfolgung von NS-Straftaten, S. 231 (Zahlen Ende 1950).

144 Gedruckt in: Um ein antifaschistisch-demokratisches Deutschland, S. 588-590.

145 DJV an Landesregierungen/Justizministerium, 20. 9. 1948, BAB, DP1 VA Nr. 6803; Melsheimer auf der Justizkonferenz vom 25./26. 11. 1948, BAB, DP1 VA Nr. 839, Bl. 13. Nach einer Rundverfügung vom 28. 10. 1948 rechnete die DJV mit einem Abschluß der meisten 201-Verfahren bis Jahresende, BAB, DP1 VA Nr. 580, Bl. 264.

146 Chefs der DJV und der DVdI an Landesregierungen/Justizministerium und Innenministerium, 29. 9. 1948, BAB, DO1/7 Nr. 424, Bl. 268.

147 Vermerk Reislers, Betr.: Maßnahmen zur baldigen Beendigung der Verfahren nach Befehl 201, 22. 6. 1949, BAB, DP1 VA Nr. 6229. 
schen Ordnung" betrachten könne ${ }^{148}$. Im Anschluß an die Regierungserklärung Grotewohls vom 12. Oktober, die die Reintegration der Masse der NSDAP-Mitglieder und Wehrmachtsoffiziere in Staat und Gesellschaft zu einem Teil des Regierungsprogramms erhoben hatte ${ }^{149}$, witterte auch die NDPD-Führung Morgenluft. Sie forderte den Ministerpräsidenten auf, neue Verfahren zur Ahndung von NS-Verbrechen nur noch aufgrund von KRG Nr. 10 einzuleiten, aber alle laufenden Strafverfahren nach Befehl Nr. 201 „gegen solche Beschuldigte oder Angeklagte, die sich keines Kriegsverbrechens oder Verbrechens gegen die Menschlichkeit schuldig gemacht haben und die nicht in den durch das Nürnberger Urteil für verbrecherisch erklärten Nazi-Organisationen in führender Stellung tätig gewesen sind", einzustellen ${ }^{150}$. Keiner dieser Wünsche ging jedoch in Erfüllung, da dies die im Antifaschismus wurzelnde Staatslegitimation der DDR in Frage gestellt hätte. Nach dem aufgrund eines Regierungsbeschlusses vom 27. Oktober gemeinsam vom Innen- und Justizministerium ausgearbeiteten, am 11. November von der Volkskammer verabschiedeten einschlägigen Gesetz erhielten NSDAP-Mitglieder und Offiziere lediglich das aktive und passive Wahlrecht zurück und durften sich in den freien Berufen, nicht aber in der Verwaltung und im Justizdienst, wieder betätigen ${ }^{151}$.

Die 201-Prozesse wurden gleichwohl fortgeführt, obwohl die Anzahl der Verfahren zur Ahndung von NS-Straftaten zurückging. Inzwischen waren auch die Untersuchungsorgane umstrukturiert worden. Aufgrund einer Anordnung Stalins vom Dezember 1948 wurde im Zuge der Vorbereitung für die Gründung eines deutschen Geheimdienstes in der SBZ die Kriminalpolizei neu organisiert; an die Stelle der K 5 trat im Herbst 1949 innerhalb der Hauptabteilung Kriminalpolizei die Abteilung C, die für die Verfolgung von NS-Straftaten das Dezernat C 10 begründete ${ }^{152}$. Zwar waren mit Weisung der Hauptverwaltung Deutsche Volkspolizei vom 17. Februar 1950 „die Bearbeitung der Vorgänge bezüglich des Befehls 201 [...] bis zum 15. März endgültig zum Abschluß zu bringen “ und die Untersuchungsorgane aufzulösen. Dies bedeutete jedoch lediglich eine Änderung des Verfahrensrechts bei den 201-Prozessen, da nun nicht länger die Polizei, sondern wieder der Staatsanwalt die Anklageschrift zu verfassen hatte ${ }^{153}$. Letztlich verfuhren die Gerichte bis 1955 bei NS-Verfahren nach Befehl Nr. 201, wobei jedoch seit 1950 die Anzahl der Prozesse und der Verurteilungen sehr stark zurückging154.

148 Vermerk Reislers, Betr.: Ergebnisse der bisherigen 201-Rechtsprechung, 26. 10. 1949, ebenda. Vgl. auch dessen Vermerk vom 4. 10. 1949, ebenda.

149 Auszug aus der Regierungserklärung Grotewohls, in: DzD II/2 (1949), S. 197.

150 Vincenz Müller und Heinrich Homann an Grotewohl, 19. 10. 1949, BAB, DP1 VA Nr. 7150.

151 Gesetz über den Erlaß von Sühnemaßnahmen und die Gewährung staatsbürgerlicher Rechte für ehemalige Mitglieder und Anhänger der Nazipartei und Offiziere der faschistischen Wehrmacht, in: GBl. 1949, S. 59 f. Zur Vorbereitung des Gesetzes siehe Vermerk Weiß, 2. 11. 1949, BAB, DP1 VA Nr. 7150.

152 Vgl. Tantzscher, In der Ostzone, S. 48 f., 52; Erler, Zur Sicherheitspolitik der KPD/SED, S. 87, gibt Oktober 1949 als Datum der Auflösung der K 5 an; Pohl, Justiz in Brandenburg (Manuskript), S. 119, geht von September aus; nach Der Staatssicherheitsdienst, S. 17, wurde die K 5 bereits im August 1949 aufgelöst.

153 MdI, HVDVP, Dienstanweisung Nr. 13/50, 17. 2. 1950, und Richtlinien zur Dienstanweisung 13/ 50 vom 17. 2. 1950, 27. 2. 1950, BAB, DP1 VA Nr. 818, Bl. 2, 4 (das zweite Dokument auch in: BStU, MfS AS 607/66, Bl. 16). Vgl. Meyer-Seitz, Verfolgung von NS-Straftaten, S. $229 f$.

154 Zahlen in: Die Haltung der beiden deutschen Staaten, S. 32. Dabei müssen jedoch von den 4092 


\section{Befehl Nr. 201 und die Anfänge der politischen Strafjustizis5}

Befehl Nr. 201 bildete nicht nur die Grundlage zur Ahndung von NS-Straftaten, sondern schuf darüber hinaus die Möglichkeit für deutsche Gerichte in der SBZ, in politischen Strafverfahren tätig zu werden. Bis zum 16. August 1947 verfügte die deutsche Justiz aufgrund der Beseitigung der Staatsschutzbestimmungen des Strafgesetzbuches durch den Kontrollrat nicht über ein entsprechendes strafrechtliches Instrumentarium. Die Besatzungsmacht behielt sich die Verfolgung politisch motivierter Straftaten selbst vor: Das Politbüro der KPdSU legte am 20. August 1946 fest, daß in politischen Fällen Verhaftungen in der SBZ allein von den Organen des sowjetischen Staatssicherheitsdienstes (MGB) vorgenommen werden sollten ${ }^{156}$; die Verhafteten wurden - wenn es überhaupt zu einem Prozeß kam - von einem Sowjetischen Militärtribunal verurteilt. Dies änderte sich mit Befehl Nr. 201. Denn indem die Vorschriften von Kontrollratsdirektive Nr. 38 den Charakter eines Strafgesetzes bekamen, wurde auch Artikel III A III im zweiten Abschnitt der Direktive justitiabel: „Aktivist ist auch", so hieß es dort, "wer nach dem 8. Mai 1945 durch Propaganda für den Nationalsozialismus oder Militarismus oder durch Erfindung und Verbreitung tendenziöser Gerüchte den Frieden des deutschen Volkes oder den Frieden der Welt gefährdet oder möglicherweise noch gefährdet." 157 Ursprünglich zur Bekämpfung von NS-Propaganda gedacht, wurde dieser Artikel mit seinen weitgefaßten Straftatbeständen zu einer der wichtigsten Grundlagen der politischen Strafjustiz in der frühen DDR.

Daß die 201-Kammern nicht nur NS-Straftäter, sondern auch "Aktivisten“ nach Artikel III A III aburteilen sollten, verdeutlichte Jakupow bereits auf der Länderkonferenz vom 14./15. November 1947 und kritisierte gleichzeitig, daß man „in der Praxis dieser Frage noch keinerlei Beachtung geschenkt" habe ${ }^{158}$. Daraufhin brachte auch Melsheimer diesem Komplex größeres Interesse entgegen. Seine Nachfrage auf der SED-Juristenkonferenz vom Januar 1948, ob in einzelnen Fällen bereits Anklage aufgrund dieses Artikels erhoben worden sei, stieß jedoch auf kein großes Echo: Ein mecklenburgischer Delegierter verneinte die Frage, und der Potsdamer Generalstaatsanwalt Ostmann gab an, lediglich über zwei derartige Fälle unterrichtet zu sein. Ostmann war sich der besonderen Bedeutung dieser Verfahren durchaus bewußt und betonte, „daß mit der Direktive [38] auch die Bekämpfung gegenwärtiger politischer Widerstände bezweckt

Verurteilten aus dem Jahre 19503324 Verurteilte der Waldheimer Prozese abgezogen werden, so daß 1950 noch 784 rechtskräftige Urteile verbleiben. Dann ist die Liste jedoch zutreffend, da sie keine Urteile nach Artikel III A III der Direktive Nr. 38 enthält: vgl. Meyer-Seitz, Verfolgung von NS-Straftaten, S. 231, Anm. 991.

155 Befehl Nr. 201 wurde nicht nur für die politische Strafjustiz, sondern auch zur kalten Enteignung von Unternehmern mißbraucht, indem diese zu NS-Verbrechern erklärt wurden. Zu diesem hier weniger interessierenden Aspekt der 201-Prozesse vgl. vor allem Weber, Justiz und Diktatur, S. $121-132$.

156 Bonwetsch, Einleitung, in: Bonwetsch/Bordjugow/Naimark, Sowjetische Politik in der SBZ, S. LIV.

157 In: Amtsblatt des Kontrollrats, 31. 10. 1946, S. 188.

158 Protokoll der Länderkonferenz am 14./15. 11. 1947, BAB, DP1 VA Nr. 6332. 
ist" ${ }^{159}$. Nach diesem mageren Ergebnis hielt die Resolution des rechtspolitischen Beirats mahnend fest: „Die Möglichkeit, die der Befehl 201 zur Bestrafung von faschistischer und militaristischer Propaganda nach Mai 1945 gibt, ist bisher in keiner Weise ausgeschöpft. Es liegen hierzu nur ganz vereinzelte Urteile mit sehr milder Strafe vor." 160

Trotz dieser eindeutigen Willensbekundung wurde auch in den nächsten Monaten diese Möglichkeit kaum genutzt, so daß Melsheimer sich genötigt sah, auf der Justizkonferenz im Juni 1948 erneut auf die „angesichts der fraglos immer noch vorhandenen und ihr Unwesen nach wie vor treibenden Anhänger des Nazismus“ zu geringe Zahl der Verfahren nach Artikel III A III hinzuweisen. Fortan strebte die DJV-Führung einen Funktionswandel der 201-Prozesse an: Während sie einerseits erkennen ließ, daß sie an einem möglichst baldigen Ende der NS-Verfahren interessiert war, ordnete sie andererseits an, nunmehr „entscheidendes Gewicht [...] auf die Verfolgung der Personen zu legen, die nach Art. III A III der Direktive 38 als Aktivisten zur Rechenschaft zu ziehen sind"161. Durch Abdruck von zwei Urteilen und einen Beschluß des Oberlandesgerichts Dresden in der „Neuen Justiz“ hoffte die DJV offensichtlich, die Gerichte zu einer möglichst weit gefaßten Auslegung des Artikels zu bewegen. In einem dieser Fälle waren antikommunistische Auslassungen bei einer Auseinandersetzung über die schlechte Ernährungssituation in der SBZ „als Propaganda für den Nationalsozialismus“ gewertet worden, während in einem Gerichtsbeschluß aufgrund einer Äußerung, derzufolge "die jetzige Polizei ,schlimmer sei als die vorhergehende“ auf eine "Bejahung der nationalsozialistischen Polizei“ geschlossen und daraus flugs ein Tatbestand nach Artikel III A III konstruiert wurde ${ }^{162}$. Auch diese Maßnahmen fruchteten nichts, so daß Melsheimer auf der Justizkonferenz vom 25./26. November 1948 feststellte, daß zu den III-A-III-Prozessen nur Sachsen „Gutes“ berichtet habe ${ }^{163}$.

Hatte man sich 1948 noch auf allgemeine Hinweise und darauf beschränkt, all diese Verfahren für berichtspflichtig zu erklären ${ }^{164}$, gingen DVdI und DJV 1949 dazu über, konkrete Anweisungen zu geben. Vorreiter war wieder die Abteilung K $5^{165}$ der Innenverwaltung, die am 21. Februar 1949 der DJV mitteilte, die Krimi-

159 Stenographische Niederschrift über die Tagung des Ausschusses für Rechtsfragen beim ZS, 3./4. 1 . 1948, SAPMO, DY 30 IV 2/1.01/70, Bl. 94, 115, $91 \mathrm{f}$. (hier auch das Zitat).

160 Stellungnahme des rechtspolitischen Beirates des Zentralsekretariats der SED am 3./4.1. 1948 zur Durchführung der Verfahren nach Befehl 201, SAPMO, DY 30 IV 2/2.1/162, TOP 4, Anlage Nr. 3.

161 Melsheimers Referat in: Die Juristenkonferenzen, S. 130; Chef der DJV an Landesregierungen/Justizministerium, 20. 9. 1948, BAB, DP1 VA Nr. 6403 (die Rundverfügung war von Melsheimer unterzeichnet).

162 Urteil des OLG Dresden, 1. 7. 1948 - 21 ERKs 130/48, in: NJ 2 (1948), S. 169-171; Beschluß des OLG Dresden, 12. 3. 1948 - 21 WS 8/48, ebenda, S. 198. Im Urteil vom 4. 5. 1948 - ERKs 109/48, ebenda, S. $197 \mathrm{f}$. hieß es: „In gleicher Weise wie gegen die Reste des Faschismus muß der Kampf geführt werden gegen jene Kräfte, die der Entfaltung der neuen Demokratie Hindernisse zu bereiten suchen durch die Äußerung und Verbreitung von Mißtrauen gegen die Ziele der neuen Demokratie und gegen ihre Organe."

${ }_{163}$ Protokoll über die 2. Juristenkonferenz in Berlin am 25./26. 11. 1948, BAB, DP1 VA Nr. 839, Bl. 13.

164 Chef der DJV an Landesregierungen/Justizministerium, 22.11. 1948, BAB, DP1 SE Nr. 937. Die Rundverfügung führte zu einer grundsätzlichen Neuregelung der Berichtspflichten.

165 Mit der Reorganisation der DVdI im Herbst 1948 wurde die Abteilung K zur Hauptabteilung und 
nalpolizei angewiesen zu haben, Personen, die aus den Westzonen stammende Zeitungen und Zeitschriften „kriegshetzerischen und antidemokratischen“ Inhalts verbreiteten, „wegen ihrer damit verbundenen Propaganda für den Krieg und die Verbreitung tendenziöser Gerüchte" nach Artikel III A III anzuklagen. Der Aufforderung an die DJV, ihrerseits eine entsprechende Anweisung an die Landesjustizministerien zu geben, wollten sich Weiß und Hirschfeld nicht verschließen; eine Rundverfügung wurde jedoch aus Gründen der deutschlandpolitischen Taktik unter Verweis auf die Pariser Außenministerkonferenz (23. Mai bis 20. Juni) zurückgestellt ${ }^{166}$. Während in diesem Fall unklar bleibt, ob die DJV tatsächlich mit einer Rundverfügung nachzog, ist dies für den nächsten Fall eindeutig erwiesen. Ende Mai 1949 ging es darum, gegen die im Zuge der Wahlen zum 3. Volkskongreß vom 15./16. Mai - die erstmals nach Einheitslisten durchgeführt worden waren - festgenommenen Personen gerichtlich vorzugehen. Die Abteilung K 5 der DVdI wies am 30. Mai alle Dezernate K 5 an, gegen Personen, „die durch Erfindung oder Verbreitung tendenziöser Gerüchte in Wort oder Schrift“ die Wahl hatten stören wollen, nach Artikel III A III Anklage zu erheben. Während dadurch $\mathrm{zwar}$ die Verbreitung westdeutscher antikommunistischer Flugblätter zu einem nach diesem Artikel zu verurteilenden Straftatbestand wurde, sollten Personen, die Wahlplakate abgerissen oder beschädigt hatten, lediglich "wegen groben Unfugs“ angezeigt und gegen diejenigen, die geäußert hatten, mit „Nein“ stimmen zu wollen, nicht weiter vorgegangen werden. Trotz einer großzügigen Haltung "gegenüber den politisch unbewußten Tätern" stand „eine energische und zielbewußte Strafverfolgung der politisch bewußten Gegner unserer Entwicklung" im Mittelpunkt der Anweisung 167. Dies hatte nichts mehr mit der Bekämpfung von NS-Propaganda zu tun. Nach einer telefonischen Rücksprache zwischen Weiß und dem K-5-Abteilungsleiter Erich Jamin übersandte auch die DJV die DVdI-Anweisung am 3. Juni an die Justizministerien und Generalstaatsanwälte der Länder ${ }^{168}$. Inwieweit diese Anweisungen befolgt wurden, ist auf zentraler Ebene nicht überliefert; aus Brandenburg sind zwölf Fälle im Zusammenhang mit den Volkskongreßwahlen bekannt, die mit Geldstrafen endeten ${ }^{169}$. Die genaue Anzahl der nach Artikel III A III vor der DDR-Gründung verurteilten Personen läßt sich anhand der MdJ-Akten nicht ermitteln; auch der zuständige Referent im Justizministerium, Reisler, verwies am 19. Oktober nur pauschal auf „einige Hundert Verfahren“, in denen fast ausschließlich Einzeltäter zu Haftstrafen zwischen ein bis drei Jahren Gefängnis verurteilt worden waren ${ }^{170}$.

Die politische Bedeutung der Prozesse nach Artikel III A III nach 1949 geht vor allem daraus hervor, daß das Ministerium für Staatsicherheit (MfS) unmittelbar nach seiner offiziellen Gründung mit Weisung vom 27. Februar 1950 dabei die

das Referat K 5 zur Abteilung K 5: vgl. Vorläufiger Geschäftsverteilungsplan der DVdI, 22. 10. 1948, BAB, DO1/7 Nr. 6, Bl. 35-39.

166 DVdI, K 5, an DJV, 21. 2. 1949, BAB, DP1 VA Nr. 6202, Bl. 1, mit handschriftlichen Vermerken von Hirschfeld (5. 3.), Weiß (4. 4.) und Reisler (10.6).

167 DVdI, Abt. K 5, an alle Dezernate K 5 der SBZ, 30. 5. 1949, BAB, DP1 VA Nr. 1110, Bl. 7.

168 DVdI, Abt. K 5, an DJV, z.H. Weiß, 31. 5. 1949 und Vermerk Weiß, 3. 6. 1949, ebenda, Bl. 6, 8.

169 Siehe Pohl, Justiz in Brandenburg (Manuskript), S. 172.

170 Vermerk Reislers, 19. 10. 1949, BAB, DP1 VA Nr. 6229. 
Untersuchungen zu führen hatte ${ }^{171}$. Wenngleich das MfS, das für diese Zwecke die Abteilung IX (seit Dezember 1953 Hauptabteilung IX ${ }^{172}$ ) bildete, in die Fußstapfen der K 5 trat, bedeutete dies nicht, daß deren Mitarbeiter weitgehend vom Staatssicherheitsdienst übernommen wurden ${ }^{173}$. Aufgrund intensiver Überprüfungen durch den MGB kamen nur etwa 10 Prozent der K 5-Mitarbeiter für den Staatssicherheitsdienst in Frage ${ }^{174}$. Die Abteilung IX, die sich in den Jahren 1950 bis 1952 hauptsächlich aus dem Personal anderer Polizeiabteilungen rekrutierte ${ }^{175}$, unterstand von 1950 bis 1956 Alfred Scholz, der sich nach seiner Gefangennahme in der Sowjetunion durch AntifaSchule, Mitarbeit im Nationalkomitee Freies Deutschland und Partisaneneinsatz für die Rote Armee bewährt und nach 1945 rasch Karriere bei der Polizei gemacht hatte ${ }^{176}$. Über wie viele Mitarbeiter die Abteilung in der ersten Hälfte der fünfziger Jahre verfügte, ist unbekannt; erst für August 1954 existieren Angaben über die Größe der Hauptabteilung IX, die damals über 297 Mitarbeiter verfügte ${ }^{177}$.

Die gerichtliche Zuständigkeit für III-A-III-Verfahren blieb von diesen Umstellungen unberührt. Als das Justizministerium in Halle im Juli 1950 auf einmal Zweifel an der Zuständigkeit der 201-Kammern in diesen Fällen hegte, wurde es vom DDR-Justizministerium eines besseren belehrt ${ }^{178}$. In der zweiten Hälfte des Jahres 1950 kristallisierte sich auch heraus, daß Strafsachen nach dem jetzt erstmals Anwendung findenden Artikel 6 der Verfassung ebenfalls von den 201-Strafkammern der Landgerichte verhandelt werden sollten. Bereits am 27. Juli 1950 hatte Weiß die Auffassung des sächsischen Justizministeriums zurückgewiesen, „daß in allen Fällen des Verstoßes gegen Artikel 6 der Verfassung das Schwurgericht zuständig sei“. In den meisten Fällen, so Weiß, werde bei einem Verstoß gegen Artikel 6 „auch der Artikel III A III der Direktive Nr. 38 verletzt sein“, und in diesen Fällen sei Anklage vor den 201-Strafkammern zu erheben ${ }^{179}$. Nachdem Weiß diese Auffassung auf einer Arbeitstagung der Generalstaatsanwälte am 29. September dahingehend präzisiert hatte, daß „bei schweren Verstößen gegen III A III, die zugleich Art. 6 der Verfassung verletzen, die Zuständigkeit der GroBen Strafkammern" gegeben sei, legte Hildegard Heinze auf der folgenden $\mathrm{Zu}$ sammenkunft am 16. November fest, daß in Verfahren nach Artikel 6 der Verfassung und Artikel III A III immer Anklage vor den 201-Strafkammern zu erheben sei ${ }^{180}$. Auch im MdJ wurde danach eine entsprechende Rundverfügung diesen Inhalts vorbereitet, die die Zuständigkeit dieser Strafkammern begründete und dar-

171 HVDVP, Abt. K, Richtlinien zur Dienstanweisung Nr. 13/50 vom 17. 2. 1950, 27. 2. 1950, BAB, DP1 VA Nr. 818, Bl. 4.

172 Vgl. Engelmann, Staatssicherheitsjustiz, S. 134.

173 So Meyer-Seitz, Verfolgung von NS-Straftaten, S. 230.

174 Vgl. Gieseke, Ministerium für Staatssicherheit, S. 374.

175 Siehe die unvollständige Aufstellung der zwischen 1950 und 1952 in der Abteilung IX der MfSZentrale eingesetzten Mitarbeiter von 1986 in: BStU, MfS HA IX 13866, Bl. 118-148.

176 Gieseke, Wer war wer im MfS, S. 64.

177 Vollnhals, Schein der Normalität, S. 216.

178 Landesregierung Sachsen-Anhalt/Justizministerium an MdJ, 11. 7. 1950, MdJ an Landesregierung Sachsen-Anhalt/Justizministerium, 27. 10. 1950, BAB, DP1 VA Nr. 6204, Bl. $2,8$.

$179 \mathrm{MdJ}$ an Landesregierung Sachsen, Justizministerium, 27. 7. 1950, BAB, DP1 VA Nr. 6200, Bl. 98.

180 Auszug aus dem Protokoll der Arbeitstagung der Generalstaatsanwälte und Oberstaatsanwälte beim Generalstaatsanwalt der DDR am 25.[sic, gemeint war 29.] 9. 1950, Arbeitstagung am 16. 11. 1950, BAB, DP3, Nr. 25, Bl. 57, 93. 
über hinaus festhielt, daß Artikel 6 der Verfassung lediglich bei schweren Fällen anzuwenden sei181. Eine solche Regelung bot sich vor allem deshalb an, weil die Richter und Schöffen dieser Kammern einer besonderen Bestätigung bedurften. Da sich die SKK als Nachfolgeeinrichtung der Sowjetischen Militärverwaltung jedoch nicht mehr darum kümmerte ${ }^{182}$, nahm das DDR-Justizministerium dieses Recht für sich in Anspruch, während bei den Schöffen die Landesjustizverwaltungen für zuständig erklärt wurden ${ }^{183}$.

Die Strafkammern nach Befehl Nr. 201, die ab Januar 1951 nur noch als „1. große bzw. kleine Strafkammern" firmierten ${ }^{184}$, wurden somit zu den für politische Strafsachen zuständigen Senaten, die nach der Gerichtsverfassung von 1952 bei den Bezirksgerichten angesiedelt waren. Ab 1951 waren neben Strafsachen nach Artikel 6 der Verfassung und Artikel III A III noch Verstöße gegen $\$ 113$ StGB (Widerstand gegen die Staatsgewalt) und Kontrollratsbefehl Nr. 2 (unerlaubter Waffenbesitz) vor den ersten Strafkammern zu verhandeln, obgleich es Anfang 1952 noch vorkam, daß solche Delikte „durch Strafbefehl erledigt oder vor dem Schöffengericht verhandelt" wurden ${ }^{185} .1951$ stellten zudem aufgrund der rapide zurückgehenden NS-Prozesse die für die Aburteilung von „Verbrechern" und „Verbrechern 2. Stufe" zuständigen kleinen Strafkammern weitgehend ihre Tätigkeit ein; im Frühjahr 1952 besaßen sie endgültig keine Existenzberechtigung mehr ${ }^{186}$. Auf die ersten großen Strafkammern konnte die DDR indes nie verzichten, boten sie doch aufgrund ihrer Besetzung und prozessualen Besonderheiten eine äußerst probate Möglichkeit zur Aburteilung politischer Straftaten.

Ein Charakteristikum der vor den ersten Strafkammern verhandelten Strafverfahren stellten insbesondere die auf den Vollmachten der K 5 aufbauenden Befugnisse des MfS im Untersuchungsverfahren dar, die nur teilweise rechtlich abgesichert waren. Ähnlich wie bei den 201-Verfahren verhafteten die Organe des MfS in eigener Regie. Bei dem anschließend beizubringenden Haftbefehl eines Haftrichters ${ }^{187}$ handelte es sich jedoch um eine Formalität. Denn die schon bei der K 5 beklagten, zahlreichen grundlosen Verhaftungen waren auch in den ersten Jahren des MfS gang und gäbe. Zwischen Januar und September 1951 etwa mußten monatlich zwischen 35 und 77 Personen wegen Mangel an Beweisen aus der Untersuchungshaft des MfS entlassen werden, so daß Staatssicherheitsminister Zaisser eine gründlichere Überprüfung des Belastungsmaterials durch die Leiter der Landesverwaltungen befahl188. Ebenfalls seit den 201-Verfahren beibehalten wurde die Praxis, unkontrolliert vom Staatsanwalt das Untersuchungsverfahren durchzuführen. „Das Aufsichtsrecht des Staatsanwalts“, so die MfS-Dienstanweisung

181 Vermerk Ganske, 28. 11. 1950, BAB, DP1 VA Nr. 7815.

182 So Heinze auf der Arbeitstagung der Generalstaatsanwälte am 28.11. 1950, BAB, DP3 Nr. 25, Bl. 93.

183 Rundverfügung Nr. 8/51, 26. 1. 1951, BAB, DP1 VA Nr. 6284, Bl. 14.

184 Ebenda.

${ }^{185}$ Siehe MdJ an SKK, 9. 8. 1951, Analyse der Rechtsprechung in Strafsachen, die sich gegen die antifaschistisch-demokratische Ordnung richten (1. Halbjahr 1951), BAB, DP1 VA Nr. 293, Bl. 1-19, insbesondere die Statistik Bl. 3.; Bericht über die Überprüfung der Gerichte und Staatsanwaltschaften Abt. I, 2. 4. 1952, BStU, MfS AS 24/55, Bl. 214.

186 So das Ergebnis der vom Politbüro eingesetzten Überprüfungskommission, ebenda.

187 MfS-Dienstanweisung Nr. 1/52, 15. 5. 1952, BStU, MfS DSt 100034.

188 MfS-Befehl Nr. 57/51, 6. 11. 1951, BStU, MfS DSt 100013. 
Nr. 1/52 vom 15. Mai 1952, „beginnt mit dem Zeitpunkt der Festnahme. Es erstreckt sich nicht auf Ermittlungshandlungen, die von den operativen Abteilungen vor der Festnahme durchgeführt wurden." ${ }^{189}$ Jedoch wurden nicht nur die geheimdienstlichen, unter den Bedingungen der Konspiration durchgeführten Ermittlungen einer justitiellen Überprüfung entzogen, sondern auch das Untersuchungsverfahren selbst. Denn die nach Abschluß der Ermittlungen dem Staatsanwalt zu übergebenden Unterlagen durften keine Hinweise „über die Arbeitsmethoden des Ministeriums für Staatssicherheit" enthalten ${ }^{190}$. Ein in den Westen geflohener Vernehmungsoffizier des MfS gab an, daß in dieser Zeit „weisungsgemäß auch alle Protokolle über die Vernehmungen des Beschuldigten entfernt [wurden], in denen dieser seine Schuld an den ihm vorgeworfenen strafbaren Handlungen bestritten hatte“191. Die Rechte des Beschuldigten im Untersuchungsverfahren unterlagen daher denselben Einschränkungen wie zu Zeiten der K 5: keine Sprech- und Schreiberlaubnis, kein Zugang zu einem Verteidiger, keine Möglichkeit, sich gegen die äußerst brutalen Vernehmungsmethoden zur Wehr zu setzen. Menschenrechtsverletzungen während der Ermittlungen des MfS waren unter diesen Bedingungen an der Tagesordnung; manche Häftlinge überlebten die Untersuchungshaft nicht ${ }^{192}$. Unter diesen Umständen war es nicht weiter verwunderlich, daß, wie das MfS nachträglich festhielt, die Bürger „das MfS mit der Gestapo und dem SD verglichen. Das Mißtrauen, welches dem Machtapparat des Faschismus entgegengebracht wurde, übertrug man auch auf das MfS. “193

Beim Gerichtsverfahren waren die formalen Befugnisse des MfS gegenüber der $\mathrm{K} 5$ in entscheidender Hinsicht verringert worden: Das MfS erstellte nicht mehr die Anklageschrift, sondern überließ dies dem aufsichtsführenden Staatsanwalt. Die sich daraus ergebenden ,Risiken' waren jedoch dadurch reduziert, daß der Generalstaatsanwalt der DDR diese Staatsanwälte nur „im Einvernehmen mit dem Ministerium für Staatssicherheit“ bestellen durfte ${ }^{194}$. Außerdem war die Praxis, den Schlußbericht des MfS unverändert als Anklageschrift zu übernehmen, weit verbreitet: „Was von den E[rmittlungs]B[ehörden] kommt“, so ein thürinigscher Staatsanwalt, „wird unterschrieben." 195 Auch wenn von der Überprüfungskommission 1952 kritisiert wurde, daß der Staatsanwalt seiner Aufgabe damit nicht gerecht wurde, änderte sich daran offensichtlich nichts. Denn auch Melsheimer forderte in der kurzen Zeit des politischen, Tauwetters“ öffentlich: „Man muß daher endlich wegkommen von der üblen Angewohnheit der Vergangenheit, daß der Staatsanwalt den Schlußbericht des Untersuchungsorgans unbesehen zur An-

${ }_{189}$ MfS-Dienstanweisung Nr. 1/52, 15. 5. 1952, BStU, MfS DSt 100034.

190 MfS-Dienstanweisung, 20. 3. 1952, in: Steuerung der Justiz im SED-Staat, S. 80-84; vgl. dazu auch Fricke/Engelmann, Konzentrierte Schläge, S. 111.

191 Aussage des Flüchtlings Bruno Krüger, 2. 12. 1953, in: Der Staatssicherheitsdienst, S. 217.

192 Siehe dazu die zahlreichen zeitgenössischen Zeugenberichte, u. a. ebenda, S. 134 f., 199f., 200-212.

Der Bericht über die Überprüfung der Gerichte und Staatsanwaltschaften Abt. I vom 2. 4. 1952

führte neun derartige Todesfälle für den Oktober 1951 allein in Mecklenburg auf (BStU, MfS AS $24 / 55, \mathrm{Bl} .223$ ).

193 Zit. nach Herbstritt, Verhältnis von Volkspolizei und MfS, S. 237.

194 MfS-Dienstanweisung Nr. 1/52, 15. 5. 1952, BStU, MfS DSt 100034; vgl. Werkentin, Politische Strafjustiz, S. 29.

195 Bericht über die Abteilung I beim Landesstaatsanwalt Thüringen, 4. 3. 1952, BStU MfS AS 24/55, Bl. 9. 
klage verdichtet und daß das Gericht seinen Eröffnungsbeschluß aus der Anklageschrift inseriert." 196

Das MfS konnte seine Einflußmöglichkeiten auch auf die Gerichtsverfahren selbst noch erweitern. Schauprozesse, „in denen vom Ministerium für Staatssicherheit bearbeitete Vorgänge zur Verhandlung kommen“, durften nur mit Genehmigung des Ministers oder des Staatssekretärs für Staatssicherheit stattfinden ${ }^{197}$. Nach Verabschiedung des neuen Gerichtsverfassungsgesetzes am 2. Oktober 1952, das grundsätzlich die Öffentlichkeit aller Verhandlungen vorschrieb, sollten die zuständigen MfS-Organe gegebenenfalls auf den Ausschluß der Öffentlichkeit beim Staatsanwalt hinwirken ${ }^{198}$. Zwar lag die Entscheidung darüber beim Gericht; aufgrund der handverlesenen Richter und Staatsanwälte dürfte es jedoch in dieser Frage kaum zu Konflikten gekommen sein. Inwieweit Staatsanwälte und Richter sich in ihrer Rechtsprechung vom MfS lenken ließen, kann im Rahmen dieser die zentralen Institutionen und Weichenstellungen thematisierenden Arbeit nicht geklärt werden. Einen Hinweis darauf, daß die Lenkung der politischen Rechtsprechung über das MfS bereits 1952 recht gut funktionierte, enthält der Überprüfungsbericht vom April 1952, der kaum Abweichungen der Gerichte von den - durch den MfS-Untersuchungsbericht weitgehend präjudizierten - Anklagen der Staatsanwälte feststellte. Abweichungen "nach unten“, wie sie bei den Landgerichten Leipzig, Chemnitz und Zwickau vorgekommen waren, führte die Kommission dann auch „auf die Tendenz des sogenannten unabhängigen Richters" zurück 199 .

Einen letzten Strang der Kontinuität zwischen den 201-Verfahren und den politischen Strafverfahren der DDR bildete die von Anfang an bestehende Neigung zur Verschleierung der politischen Dimension der Tätigkeit der 201-Kammern. Unmittelbar nach ihrer Begründung im Herbst 1947 wurden sie in der Bevölkerung, aber auch in der Justiz als "politische Strafkammern“ bezeichnet. Mielke kritisierte diese Begrifflichkeit mehrfach, unterstellte, „daß diese Benennung in Thüringen kein Zufall ist“, und forderte die SED-Richter und -Staatsanwälte dazu auf, dem entgegenzuwirken ${ }^{200}$. Melsheimer formulierte sehr offen auf der SEDJuristenkonferenz vom Januar 1948: „Natürlich besteht kein Zweifel daran, daß [die] Strafsachen in der Wurzel politische Sachen sind. Aber diese Bezeichnung ,politische Strafkammer" ist ein Fehler." 201 Zwar ging es damals noch nicht primär um die Verurteilung politischer Strafsachen aus der Nachkriegszeit, sondern um die strafrechtliche Bewertung politischen Verhaltens vor 1945; vor dem Hintergrund der politischen Instrumentalisierbarkeit der Verfahren und dem Bedürfnis, sich von der NS-Sondergerichtsbarkeit eindeutig abzugrenzen, bestanden die

196 Bericht über die Überprüfung der Gerichte und Staatsanwaltschaften Abt. I, 2. 4. 1952, ebenda, Bl. 201; Melsheimer, Sozialistische Gesetzlichkeit im Strafverfahren, S. 294.

197 MfS Berlin an Bezirksverwaltungen, 23. 9. 1952, BStU, MfS DSt 101161.

198 Dienstanweisung Nr. 38/53, 1. 12. 1953, BStU, MfS DSt 100880.

199 Bericht über die Überprüfung der Gerichte und Staatsanwaltschaften Abt. I, 2. 4. 1952, BStU, MfS AS 24/55, Bl. 205, 211 (hier das Zitat).

200 So Mielke auf der Länderkonferenz vom 14./15. 11. 1947, BAB, DP1 VA Nr. 6332, und auf der 3. Tagung des Ausschusses für Rechtsfragen beim ZS, 3./4. 1. 1948, SAPMO, DY 30 IV 2/1.01/70, Bl. 109 (hier das Zitat).

201 Ebenda, Bl. 126. 
SED-Juristen jedoch auf der Bezeichnung: „Strafkammern nach Befehl Nr. 201“. Auch die DJV arbeitete, ohne daß es einer Aufforderung der DVdI bedurfte, nur mit dieser Begrifflichkeit ${ }^{202}$. Als ab dem Januar 1951 per Rundverfügung des $\mathrm{MdJ}$ auch noch der Zusatz „gemäß Befehl 201“ gestrichen wurde und nur noch von der "1. großen bzw. kleinen Strafkammer" 203 die Rede sein durfte, war es nur noch ein kleiner Schritt zu der Weisung vom 5. September, derzufolge die Bezeichnung "Politische Häftlinge" unstatthaft sei, da in der DDR "niemand seiner Gesinnung wegen inhaftiert" werde 204 . Zur politischen Strafjustiz in der SBZ/DDR gehörte mithin seit ihren Anfängen eine an George Orwells „Newspeak“ erinnernde sprachliche Verharmlosung ihrer Zweckbestimmung.

Insgesamt kann die Bedeutung von SMAD-Befehl Nr. 201 für die Transformation des Justizwesens in der SBZ/DDR gar nicht hoch genug eingeschätzt werden. Denn mit der sowjetischen Entscheidung, in der Entnazifizierungsfrage die gemeinsame Linie der Alliierten zu verlassen und in der SBZ einen eigenen Weg einzuschlagen, wurden erstmals in nachhaltiger Weise sowjetische Praktiken auf Polizei und Justiz übertragen. Ungeachtet früherer Ansätze markiert Befehl Nr. 201 somit den Beginn der Sowjetisierung der Verhältnisse in der politischen Strafjustiz. Die Besatzungsmacht sah zudem in der Durchführung des Befehls durch die deutschen Justizorgane einen Testfall, der zeigen sollte, ob sich diese als ebenso getreue Vollstrecker ihres Willens erweisen würden wie die Polizei. Für die SED, die damals begann, ihren Führungsanspruch im Justizwesen zu reklamieren, war dies eine willkommene Gelegenheit, um zu demonstrieren, daß die SMAD sich bei der Bestrafung von NS-Straftätern auf sie verlassen konnte. Um sich entsprechend profilieren zu können, mußte sie sich erstmals auch in der Justizsteuerung engagieren. Sowohl für die sowjetische Besatzungsmacht als auch für die Einheitspartei erwies sich dabei die Innenverwaltung als weitaus wichtiger als die Justizverwaltung, die erhebliche Kompetenzeinbußen hinnehmen mußte. Dies galt für die Ausarbeitung der Ausführungsbestimmungen genauso wie für die Lenkung der Rechtsprechung: Im letzteren Fall fungierte sie ausschließlich als Übermittlerin von Anweisungen der SMAD oder der Innenverwaltung. Im Zuge dieses Prozesses ließ die DJV, in der 1948 die Führungebene mit SED-Genossen besetzt wurde, die Aushöhlung von rechtsstaatlichen Grundsätzen mehr und mehr zu. Die Versuche der SED, die Justiz über die Parteischiene zu steuern, beschränkten sich im wesentlichen auf die Jahreswende 1947/48 und waren in dieser Zeit, als die Prozesse gerade anliefen, noch wenig erfolgreich. Dies änderte sich im Verlauf des Jahres 1948, als sie stärker durch den Justizapparat wirken konnte und nach dem Abschluß des dritten Volksrichterlehrgangs sehr viel mehr SED-Richter und -Staatsanwälte zur Verfügung standen. Auch wenn die Gerichte in den 201-Prozessen den Anweisungen zu harten Bestrafungen nicht durchgehend Folge leiste-

202 Vermerk Winkelmann, 13. 2. 1948, BAB, DP1 VA Nr. 6204, Bl. 99; DJV an DVdI, 30. 9. 1948, BAB, DP1 VA Nr. 6200, Bl. 65. Vorangegangen war einen Aufforderung der DVdI vom 24.8. 1948, alle Gerichte davon in Kenntnis zu setzen, daß die Bezeichnung "Große Politische Strafkammer" unstatthaft sei, ebenda, Bl. 63.

203 Rundverfügung Nr. 8/51, 26. 1. 1951, BAB, DP1 VA Nr. 6284, Bl. 14.

204 Rundverfügung Nr. 125/51, 5. 9. 1951, in: Unrecht als System, Bd. 1, S. 34. 
ten, wurden in den Jahren 1948/49 doch erstmals eine Reihe von Steuerungsmechanismen erprobt, auf die auch später zurückgegriffen werden sollte.

Für die politische Strafjustiz der frühen DDR waren die Verfahren nach Befehl Nr. 201 in dreierlei Hinsicht von großer Bedeutung: erstens durch die unpräzise Bestimmung des Artikels III A III der Kontrollratsdirektive Nr. 38, die eine der DDR-typischen Blankettstraftatbestände lieferte, zweitens durch die weit gefaßten Kompetenzen der politischen Polizei, die de facto auf das MfS übergingen, und drittens durch die Sonderstrafkammern, die zur Aburteilung vermeintlicher oder tatsächlicher politischer Gegner des Regimes beibehalten wurden und deren Existenz dem auch in der DDR-Verfassung niedergelegten Verbot von Sondergerichten widersprach. Das MdJ schließlich trat im Hinblick auf seine untergeordnete Rolle bei der Steuerung der politischen Strafjustiz in die Fußstapfen der DJV bei der Lenkung der 201-Verfahren: Es fungierte nie als Initiator, sondern höchstens als Übermittler anderer Steuerungszentren bei entsprechenden Justizkampagnen und mußte zudem hinnehmen, daß es sehr viel weniger effektiv als andere Instanzen die Prozesse vor Ort beeinflussen konnte. 